\title{
The BESIII Physics Programme
}

\author{
Chang-Zheng Yuan ${ }^{1,2}$ and Stephen Lars Olsen ${ }^{2}$ \\ ${ }^{1}$ Institute of High Energy Physics, Chinese Academy of Sciences, Beijing 100049, China \\ ${ }^{2}$ University of Chinese Academy of Sciences, Beijing 100049, China
}

(Dated: July 29, 2019)

\begin{abstract}
The standard model of particle physics is a well-tested theoretical framework, but there are still a number of issues that deserve further experimental and theoretical investigation. For quark physics, such questions include: the nature of quark confinement, the mechanism that connects the quarks and gluons of the standard model theory to the strongly interacting particles; and the weak decays of quarks, which may provide insights into new physics mechanisms responsible for the matter-antimatter asymmetry of the Universe. These issues are addressed by the Beijing Spectrometer III (BESIII) experiment at the Beijing ElectronPositron Collider II (BEPCII) storage ring, which for the past decade has been studying particles produced in electron-positron collisions in the tau-charm energy-threshold region, and has by now accumulated the world's largest datasets that enables searches for nonstandard hadrons, weak decays of the charmed particles, and new physics phenomena beyond the standard model. Here, we review the contributions of BESIII to such studies and discuss future prospects for BESIII and other experiments.
\end{abstract}




\section{INTRODUCTION}

The two main questions addressed by particle physics are "what are the most elementary building blocks of matter?' and 'what are the forces between them?'. In the standard model (SM) of particle physics, the well-established theoretical description of the fundamental particles and their mutual interactions, the building blocks are quarks and leptons, and the forces between them are mediated by the exchange of gauge bosons associated with the weak, electromagnetic, and strong interactions [1]. Although the SM has been successfully tested by numerous experiments, many of its detailed predictions remain to be confirmed (for example, the existence of nonstandard hadrons and the quark mixing properties in weak interaction), and substantial theoretical and experimental efforts are currently being devoted to this end.

Particle accelerators and experiments specifically aimed at studies of these issues include: the BaBar [2] and Belle [3] experiments at the PEPII and KEKB $B$-factory colliders operating during 1999-2008 and 1999-2010, respectively; the currently operating BESIII [4] experiment at the BEPCII electron-positron collider and the $\mathrm{LHCb}$ [5] experiment at the high energy Large Hadron Collider (LHC) proton-proton collider; the Belle II/SuperKEKB project [6] that is now starting to take data; and proposed electron-positron 'super tau-charm' factories: STCF in Hefei, China [7] and SCTF in Novosibirsk, Russia [8].

In the early 2000s, the BaBar and Belle experiments established that the experimentally observed $C P$ violations [9] in $B \bar{B}$ system can be explained as consequences of an irreducible complex phase in the Cabibbo-Kobayashi-Maskawa (CKM) six-quark flavour-mixing matrix, as first suggested in Ref. [10]. In addition, these experiments discovered a number of nonstandard hadronic states [11-15] with properties that indicate their substructures are more complex than the quark-antiquark mesons and three-quark baryons of the conventional quark model [16, 17].

The BESIII experiment [4] (see Appendix A for a short description), the third (and current) phase of a thirty-years-old research program based at the Institute of High Energy Physics in Beijing, investigates both hadronic states and weak decay of charmed quark with electron-positron $\left(e^{+} e^{-}\right)$collision data in the center-of-mass energy $\left(\mathrm{E}_{\mathrm{CM}}\right)$ range between 2.0 and $4.6 \mathrm{GeV}$ produced by the BEPC collider, with a luminosity that started at $10^{29} \mathrm{~cm}^{-2} \mathrm{~s}^{-1}$ at the Beijing Spectrometer in 1988 [18], improved to $10^{31} \mathrm{~cm}^{-2} \mathrm{~s}^{-1}$ with BESII in 2002 [19], and, with BESIII and the BEPCII collider, reached $10^{33} \mathrm{~cm}^{-2} \mathrm{~s}^{-1}$ in 2016 . In 2008, the single-ring BEPC collider, in which the counter-rotating $e^{+}$and $e^{-}$beams shared the same magnets and vacuum chamber, was replaced by BEPCII [20], a high performance, two-ring $e^{+} e^{-}$collider, in which each beam has its own magnet and vacuum systems. At the same time, BESII was replaced by BESIII, a state-of-the-art detector with substantially improved capabilities.

The $2 \sim 4.6 \mathrm{GeV}$ center-of-mass $(\mathrm{CM})$ energy region covers the thresholds for the production of pairs of particles that contain charm-quarks $(c)$ and charm-antiquarks $(\bar{c})$ and includes the narrow charmonium $(c \bar{c})$ resonances $J / \psi$ and $\psi(3686)$, which are prolific sources of hadrons comprising of the up $(u)$, down $(d)$ and strange $(s)$ light quarks. The cross section for $D^{0}(c \bar{u})$ and $D^{+}$

$(c \bar{d})$ mesons and their antiparticles has a maximum at $3.77 \mathrm{GeV}$, the peak of the $\psi(3770)$ resonance, and large samples of $D_{s}(c \bar{s})$ mesons are produced at the nearby $\psi(4160)$ resonance. The energy threshold for pair production of the $\Lambda_{c}(c u d)$, the lightest baryon that contains a charm quark, is $\mathrm{E}_{\mathrm{CM}}=4.573 \mathrm{GeV}$, and also accessible to BEPCII. In addition, this energy region includes the thresholds for $\tau$-lepton and all of the stable hyperons (baryons that contain one or more $s$-quarks). Studies at the near-threshold energy for the production and decays of these particles have a number of unique advantages (large production rate, clean environment, very low background, high 
detection efficiency, and so on) over measurements done at higher energies. As a result the BES program addresses a diverse range of interesting physics with unprecedented sensitivity.

Notable achievements of the BES and BESII phases of this program include: BES's first result, which was a five-fold improvement in the precision of the $\tau$-lepton's measured mass that was two standard deviations lower than the previous world average value at that time and cleared up a discrepancy with SM expectations [21]; the first observation of the purely leptonic $D_{s}^{+} \rightarrow \mu^{+} \nu$ decay process [22]; precision measurements of the annihilation cross section for $e^{+} e^{-} \rightarrow$ hadrons that provided essential inputs to the SM predictions for the Higgs boson mass and the anomalous magnetic moment of the muon, $(g-2)_{\mu}[23]$; the discoveries of the $\sigma(500)$ [24] and $\kappa(700)$ [25] scalar mesons; and a comprehensive study of the so-called $\rho \pi$ puzzle in vector charmonium decays [26].

During its first ten years of operation, BESIII accumulated the world's largest data sets of $D$ and $D_{s}$ meson decays, 10 billion $J / \psi$ and 450 million $\psi(3686)$ events, and about 100 million events with $\mathrm{E}_{\mathrm{CM}}$ between 4 and $4.6 \mathrm{GeV}$ for studies of nonstandard hadrons and the $\Lambda_{c}$ baryon. BESIII data provide stringent constraints on the CKM quark-flavour mixing scheme with precision measurements of the $\left|V_{c s}\right|$ and $\left|V_{c d}\right|$ CKM matrix elements that modify the strengths of weak interaction $c \rightarrow s$ and $c \rightarrow d$ quark transitions, and strong-interaction phases in $D$-meson decays. These strong phases are basic quantities that are essential inputs to other experiments that determine $\gamma$, the $C P$-violating complex phase angle of the $V_{u b} \mathrm{CKM}$ matrix element responsible for $B$-meson decays. The CKM matrix elements are fundamental constants of the SM that have to be measured in experiment. Their values are strictly constrained by unitarity; any deviation from unitarity would be an unambiguous signal for new, non-SM physics.

In the conventional quark model, mesons comprise of one quark and one antiquark, whereas baryons comprise of three quarks. This simple picture successfully describes almost all of the hadrons that were observed prior to the operation of the BaBar and Belle $B$-factory experiments. However, other, nonstandard configurations have been proposed since the very inception of the quark model [16, 17]. Although these were the subject of a long series of experimental searches [27], the results were inconclusive. However, starting in 2003, Belle and BaBar discovered a number of meson states that decay to final states that contain both a $c$ - and a $\bar{c}$-quark [9]. Whereas some of these states have properties that fit well the expectations for the conventional $c \bar{c}$ mesons of the charmonium model [28], others have properties that do not match those of any $c \bar{c}$ meson and can only be accommodated by nonstandard, multi-quark configurations [11,-15, 29]. These latter charmonium-like states are collectively referred to as the XYZ mesons to indicate that their underlying structure is still not well understood. During the same time period, the Belle group discovered candidates for nonstandard mesons in the bottom-quark sector [30] and the LHCb group found strong candidates for five-quark (pentaquark) baryons [31].

With the capability of adjusting the $e^{+} e^{-} \mathrm{CM}$ energy to the peaks of resonances and to justbelow and just-above the energy thresholds for particle-antiparticle pair formation, combined with the clean experimental environments due to near-threshold operation (production of one additional hadron needs to increase the CM energy by more than $100 \mathrm{MeV}$ ), BESIII is uniquely able to perform a broad range of critical measurements of the weak decays of strange and charmed particles, and the production and decays of many of the nonstandard XYZ meson states. Here, we briefly review some highlights of the BESIII program, including: precision measurements of CKM matrix elements; studies of charmed particle decays; discoveries of new XYZ mesons; in-depth investigations of light hadrons; refined measurements of the fundamental properties of baryons; contributions to stringent tests of the SM, such as the anomalous muon magnetic moment $(g-2)_{\mu}$, with precision measurements of the cross section for $e^{+} e^{-}$annihilation into hadrons; and preci- 
sion measurements of the production cross-sections and decay properties of hyperons. We present our perspective on the potential future results from BESIII and other experiments, and discuss the opportunities for studies at proposed next-generation facilities.

\section{MEASUREMENT IMPROVEMENTS OF $m_{\tau}$ AND $\sigma\left(e^{+} e^{-} \rightarrow\right.$ hadrons $)$}

\section{A. Three decades of $m_{\tau}$ measurements}

The $\tau$-lepton mass $\left(m_{\tau}\right)$ is a fundamental parameter of the SM and a precise knowledge of its value is essential for tests of the lepton flavour universality (LFU). The current experimental precision is primarily due to a $2014 \mathrm{BESIII}$ measurement $m_{\tau}=1776.91 \pm 0.18 \mathrm{MeV} / c^{2}$ [32] that is in good agreement with the original $1992 \mathrm{BES}$ value: $1776.9 \pm 0.5 \mathrm{MeV} / \mathrm{c}^{2}$ [21], but with significantly better precision. The BESIII measurement benefited from the implementation of a new laser backscattering beam energy measurement system, BEMS [33].

This level of precision is still three orders of magnitude poorer than that for the muon-lepton mass $m_{\mu}$ and continued improvements are needed. With refinements of the BEMS and more data near the energy threshold for $\tau^{+} \tau^{-}$production, BESIII will further improve the precision of this measurement to $\pm 0.10 \mathrm{MeV} / c^{2}$, and maintain the program's leading role in this direction [34].

\section{B. Precision measurement of vacuum polarization of virtual photons}

The measured value of $(g-2)_{\mu}$ from Brookhaven National Laboratory experiment E821 [35] is $~ 3.5$ standard deviations higher than the SM prediction [36, 37], a discrepancy that has inspired elaborate follow-up experiments at Fermilab [38] and J-PARC [39]. The SM prediction for $(g-$ $2)_{\mu}$ is very sensitive to the effects of hadronic vacuum polarization (HVP) of the virtual photon, which are about 100 times larger than the current experimental uncertainty and, thus, must be determined with high precision. Vacuum polarization also has a critical influence on precision tests of the electroweak theory, which rely on a precise knowledge of $\alpha(s)$, the running quantum electrodynamics (QED) coupling constant. Because of vacuum polarization, $\alpha^{-1}\left(m_{Z}^{2}\right)=128.95 \pm$ 0.01 [36] is about 6\% below its long-distance value of $\alpha^{-1}(0)=137.04$. About half of this difference is due to HVP.

Since HVP effects are non-perturbative, they cannot be directly computed from first principles. Recent computer-based lattice quantum chromodynamics (LQCD) calculations have made significant progress, but the uncertainties are still large [40,41]. Instead, the most reliable determinations of the HVP contributions to $(g-2)_{\mu}$ and $\alpha\left(m_{Z}^{2}\right)$ use dispersion relations with input from experimental measurements of cross-sections for the $e^{+} e^{-}$annihilation into hadrons [36, 37]. The data used for the most recent determinations are mostly from the SND [42], BaBar [43], BESIII [44], CMD-2 [45, 46], and KLOE [47] experiments. BaBar and KLOE operations have been terminated (although the data analysis continues), leaving SND, CMD-3 [48], and BESIII as the only running facilities with the capability to provide the improvements in precision that will be essential for the evaluation of $(g-2)_{\mu}$ with a precision that will match that of the new experimental measurements at Fermilab [38] and J-PARC [39].

With data taken at $\mathrm{E}_{\mathrm{CM}}=3.773 \mathrm{GeV}$ (primarily for studies of $D$-meson decays) BESIII measured the cross-sections for $e^{+} e^{-} \rightarrow \pi^{+} \pi^{-}$, where $\pi^{+}$and $\pi^{-}$denote pions, at $\mathrm{E}_{\mathrm{CM}}$ between 
0.6 and $0.9 \mathrm{GeV}$ [44], which covers the $\rho \rightarrow \pi^{+} \pi^{-}$peak, where $\rho$ denotes a rho meson, the major contributor to the HVP dispersion relation integral. These measurements used initial state radiation (ISR) events in which one of the incoming beam particles radiates a $\gamma$-ray with energy $E_{\mathrm{ISR}}=x \mathrm{E}_{\mathrm{CM}} / 2$ before annihilating at a reduced $\mathrm{CM}$ energy of $\mathrm{E}_{\mathrm{CM}}^{\prime}=\sqrt{1-x} \mathrm{E}_{\mathrm{CM}}$. The relative uncertainty of the BESIII measurements is $0.9 \%$, which is similar to the precision of the BaBar [43] and KLOE [47] results. The BESIII measured values agree well with the KLOE results for energies below $0.8 \mathrm{GeV}$, but are systematically higher at higher energies. In contrast, the BESIII results agree with BaBar at higher energies, but are lower at lower energies. The detailed comparisons are shown in Fig. 1. Nevertheless, the contributions of $e^{+} e^{-} \rightarrow \pi^{+} \pi^{-}$to the $(g-2)_{\mu}$ HVP calculation from these experiments are overall in agreement within two standard deviations, and the observed $\sim 3.5$ standard deviation difference between the calculated muon magnetic moment value and the E821 experimental measurement persists.

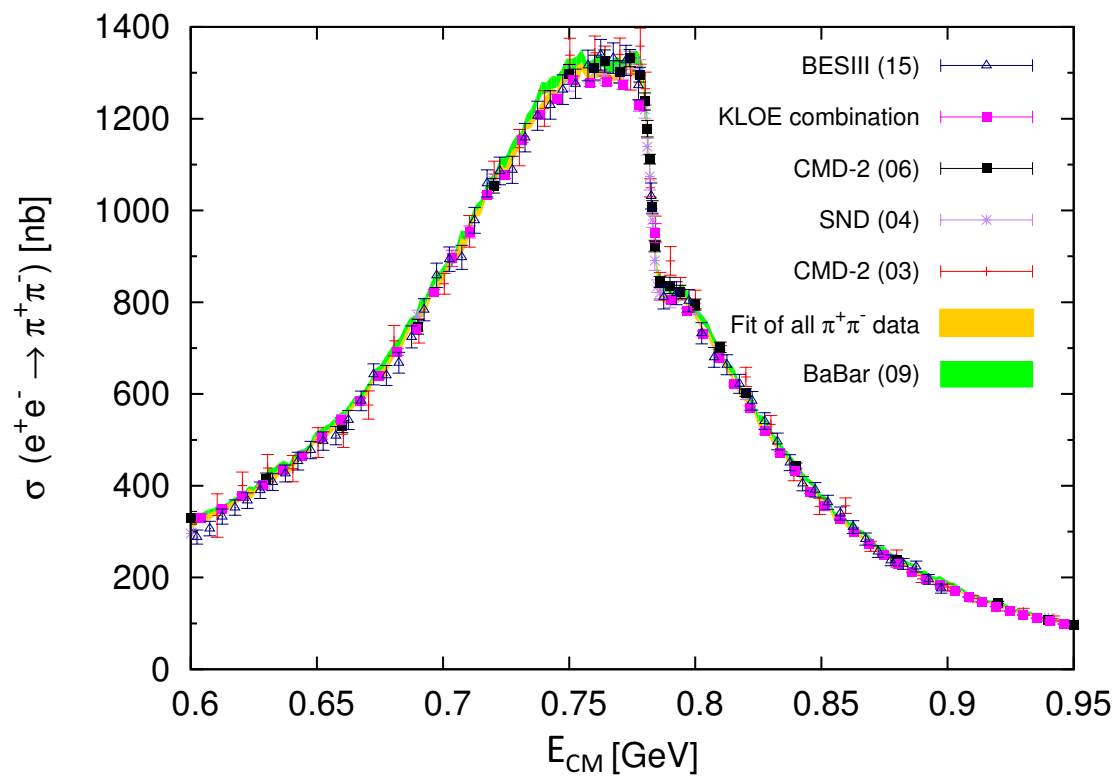

FIG. 1: Measurements of cross-section $\sigma\left(e^{+} e^{-} \rightarrow \pi^{+} \pi^{-}\right)$from SND [42], CMD-2 [45, 46], BaBar [43], KLOE [47], and BESIII [44]. The structure near $\mathrm{E}_{\mathrm{CM}}=0.78 \mathrm{GeV}$ is due to interference between $\rho \rightarrow$ $\pi^{+} \pi^{-}$and $\omega \rightarrow \pi^{+} \pi^{-}$; the orange band is the result of a fit from Ref. [37]. The numbers in parentheses in the figure legend denote the year when the respective reference was published. Reproduced from Ref. [37].

In the ISR process, the direction of the radiated $\gamma$-ray is strongly peaked in the beam direction and does not usually register in the detector. In the BESIII measurements reported in Ref. [44], the $\gamma$-ray had to be detected, which limited the usable data sample to only a small fraction of the produced events. With larger data sets, not only will these measurements have improved statistical precision, but, by including events in which the $\gamma$-ray is emitted along or very close to the beam direction and undetected, but with its presence inferred from energy-momentum constraints, cross sections at even lower $\mathrm{E}_{\mathrm{CM}}^{\prime}$ values will be measured. In addition, BESIII is currently measuring cross sections for other modes, such as $e^{+} e^{-} \rightarrow \pi^{+} \pi^{-} \pi^{0}$ [49], $2\left(\pi^{+} \pi^{-}\right)$, and $\pi^{+} \pi^{-} 2 \pi^{0}$, that also contribute to the HVP integral, albeit at a lower level [50]. For $\mathrm{E}_{\mathrm{CM}}$ above $2 \mathrm{GeV}$, BESIII does not need to use the ISR method and can make higher precision, direct cross-section measurements [51]. These efforts will continue to improve our understanding of the vacuum polarization of the photon and provide essential input to other experimental programs. 


\section{PRECISION MEASUREMENTS OF CHARMED PARTICLE DECAYS}

The charm quantum number is conserved by the strong and electromagnetic forces. As a result the lightest charmed hadrons, the $D^{0}, D^{+}$, and $D_{s}^{+}$mesons, and the $\Lambda_{c}^{+}$baryon, decay via weak interactions. The rates and dynamics of these decays are sensitive to the strong interactions between the parent charm and light quarks and those between the final-state light quarks. The strengths of the weak decays are proportional to the Fermi constant $G_{F}$ multiplied by the $V_{c d}$ and $V_{c s}$ CKM matrix elements. In principle, the strong interactions between quarks can be computed by quantum chromodynamics (QCD), but the calculations are extremely difficult. Instead, QCD-inspired models, or LQCD calculations are used. Rapid advances in the latter need to be rigorously tested by measurements of purely leptonic and semileptonic $D$ and $D_{s}$ meson decays with improved precision.

\section{A. Purely leptonic decays}

The rates for purely leptonic charmed meson decays, $D_{q}^{+} \rightarrow \ell^{+} \nu$, where $q=d$ or $s$ and $\ell=e$, $\mu$, or $\tau$, electron, muon, or tau, respectively, and $\nu$ stands for neutrino, are proportional to the product of $\left|V_{c q}\right|$, the relevant CKM matrix element, and $f_{D_{q}^{+}}$, the $D_{q}^{+}$-meson decay constant. In terms of these parameters, the SM decay width is given by

$$
\Gamma\left(D_{q}^{+} \rightarrow \ell^{+} \nu\right)=\frac{G_{F}^{2} f_{D_{q}^{+}}^{2}}{8 \pi}\left|V_{c q}\right|^{2} m_{\ell}^{2} m_{D_{q}^{+}}\left(1-\frac{m_{\ell}^{2}}{m_{D_{q}^{+}}^{2}}\right)^{2},
$$

where $m_{\ell}$ is the lepton mass, and $m_{D_{q}^{+}}$is the $D_{q}^{+}$-meson mass, which are both well measured. Thus, the determination of $\Gamma\left(D_{q}^{+} \rightarrow \ell^{+} \nu\right)$ directly measures the product $\left|V_{c q}\right| f_{D_{q}^{+}}$.

In principle, the $f_{D_{q}^{+}}$values can be computed using LQCD. At present, the obtained precision for these calculations are at the part per thousand level [52], and can translate leptonic decay-rate measurements into high-precision determinations of the $\left|V_{c q}\right|$ CKM matrix elements.

The $\psi(3770)$ resonance is only $30 \mathrm{MeV} / c^{2}$ above the $D \bar{D}$ mass threshold and predominantly decays to final states with a $D \bar{D}$ meson pair and nothing else. Thus, when a final-state $D$ (or $\bar{D}$ ) meson is fully reconstructed in one of its common hadronic decay modes, the accompanying $\bar{D}$ $(D)$ is tagged, meaning that it must be the parent of all the remaining particles in the event and its four-momentum is specified. When a tagged $D^{+}$decays to $\mu^{+} \nu$, the mass of the (undetected) zero-mass neutrino can be inferred from energy-momentum conservation. This tagging feature is a powerful tool for charmed-particle decay measurements that is only possible in near-threshold experiments.

In a sample of 1.7 million tagged $D^{ \pm}$mesons from $\psi(3770) \rightarrow D^{+} D^{-}$decays, BESIII found $409 \pm 21 D^{ \pm} \rightarrow \mu^{ \pm} \nu$ signal events over a small background (see Fig. 2), corresponding to the world's best branching fraction measurement: $\mathcal{B}\left(D^{+} \rightarrow \mu^{+} \nu\right)=(3.71 \pm 0.20) \times 10^{-4}$ [53], which translates to $f_{D^{+}}\left|V_{c d}\right|=(45.8 \pm 1.3) \mathrm{MeV}$. This result, in conjunction with the CKM matrix element $\left|V_{c d}\right|$ determined from a global SM fit [54], implies a value for the weak decay constant $f_{D^{+}}=203.9 \pm 5.6 \mathrm{MeV}$. Alternatively, using this result with an LQCD calculated value for $f_{D^{+}}(212.7 \pm 0.6 \mathrm{MeV})$ [52], one finds $\left|V_{c d}\right|=0.2151 \pm 0.0060$. In either scenario, these are the most precise results for these quantities to date. 

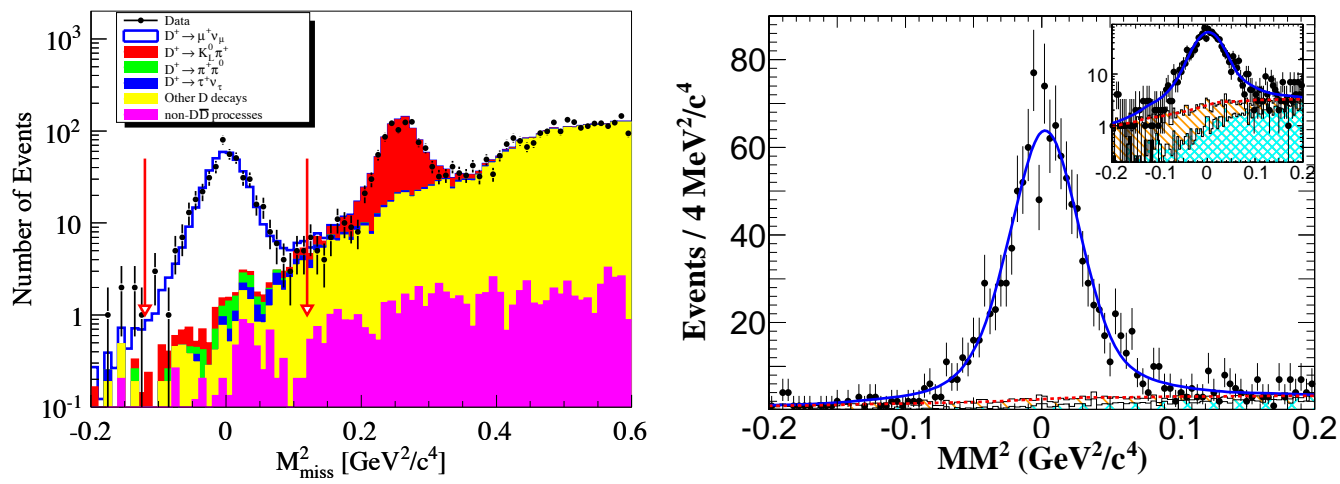

FIG. 2: The peaks centered at the square of the invariant mass of the missing particles $M_{\text {miss }}^{2}=0$ are the signals for the purely leptonic decays $D^{+} \rightarrow \mu^{+} \nu$ [53] (left panel) and $D_{s}^{+} \rightarrow \mu^{+} \nu$ [55] (right panel) measured at BESIII. Red arrows in the left panel are the boundaries of the signal region, and the inset in the right panel shows the same distribution in log scale.

BESIII reported a measurement of the absolute decay branching fractions $\mathcal{B}\left(D_{s}^{+} \rightarrow \mu^{+} \nu\right)$ with 0.39 million tagged $D_{s}^{+}$mesons produced via $e^{+} e^{-} \rightarrow D_{s}^{+} D_{s}^{*-}$ at $\mathrm{E}_{\mathrm{CM}}=4.178 \mathrm{GeV}$ [55]. In this report, the inclusion of charged conjugate states is always implied; for example, $D_{s}^{+} D_{s}^{*-}$ indicates both $D_{s}^{+} D_{s}^{*-}$ and $D_{s}^{*+} D_{s}^{-}$. The $1136 \pm 33 D_{s}^{ \pm} \rightarrow \mu^{ \pm} \nu$ signal events (see Fig. 2) were used to determine $\mathcal{B}\left(D_{s}^{+} \rightarrow \mu^{+} \nu\right)=(5.49 \pm 0.22) \times 10^{-3}$, which corresponds to $f_{D_{s}^{+}}\left|V_{c s}\right|=$ $246.2 \pm 5.0 \mathrm{MeV}$. If $\left|V_{c s}\right|$ is fixed at its latest global SM fit value [54], the $D_{s}^{+}$decay constant is determined to be $f_{D_{s}^{+}}=252.9 \pm 5.1 \mathrm{MeV}$. Alternatively, fixing $f_{D_{s}^{+}}$at its value from LQCD calculations $(249.9 \pm 0.4 \mathrm{MeV})$ [52, 56], yields $\left|V_{c s}\right|=0.985 \pm 0.020$. Either of these measurements qualify as the currently most precise value.

In principle, $D_{q}^{+} \rightarrow e^{+} \nu$ and $\tau^{+} \nu$ can also be measured to obtain the same decay constantCKM matrix element product. According to Eq. (1), the expected relative decay widths for the $\tau^{+} \nu, \mu^{+} \nu$, and $e^{+} \nu$ modes are $2.67: 1: 2.35 \times 10^{-5}$ for $D^{+}$and $9.75: 1: 2.35 \times 10^{-5}$ for $D_{s}^{+}$; the $\mathrm{SM} D_{q}^{+} \rightarrow e^{+} \nu$ decay widths are highly suppressed by helicity conservation, and $2 \sim 3$ orders of magnitude below the sensitivity of BESIII. Although the SM $D_{q}^{+} \rightarrow \tau^{+} \nu$ decay widths are larger than those for $D_{q}^{+} \rightarrow \mu^{+} \nu$, the presence of additional final-state neutrinos from the $\tau$ decay voids the kinematic constraint (the inference of the four-momentum of a single missing neutrino from measurements of all the accompanying particles in the event) that is available for $\mu^{+} \nu$ and this results in more background. Nevertheless, $D_{s}^{+} \rightarrow \tau^{+} \nu$ decay is currently being studied at BESIII with an expected result that will have a precision comparable to that achieved for $D_{s}^{+} \rightarrow \mu^{+} \nu$. This result should improve the accuracy of the $\left|V_{c s}\right| f_{D_{s}^{+}}$measurement, and can also be used to test LFU.

\section{B. Semileptonic decays}

Semileptonic decay rates, in conjunction with form factors determined from LQCD calculations, provide independent measurements of the $\left|V_{c s}\right|$ and $\left|V_{c d}\right|$ CKM matrix elements. The first high-precision, semi-leptonic decay rate measurements were made by CLEO-c based on data sets accumulated at the $\psi(3770)$ resonance peak [57]. BESIII, with triple the amount of $\psi(3770)$ data, significantly improved the measurements of these quantities, thereby keeping pace with improve- 
ments in the relevant LQCD results.

The most relevant measurements are for the $D^{0} \rightarrow K^{-} \ell^{+} \nu$ and $\pi^{-} \ell^{+} \nu$, and $D^{+} \rightarrow \bar{K}^{0} \ell^{+} \nu$ and $\pi^{0} \ell^{+} \nu$ decay channels, with $\ell=e$ or $\mu$ and $K$ standing for kaons. With 2.8 million tagged $D^{0}$ mesons, $70727 \pm 278 D^{0} \rightarrow K^{-} e^{+} \nu$ and $6297 \pm 87 D^{0} \rightarrow \pi^{-} e^{+} \nu$ signal events are observed, and absolute decay branching fractions are determined to be $\mathcal{B}\left(D^{0} \rightarrow K^{-} e^{+} \nu\right)=(3.505 \pm 0.035) \%$ and $\mathcal{B}\left(D^{0} \rightarrow \pi^{-} e^{+} \nu\right)=(0.295 \pm 0.005) \%$ [58]. From differential decay rate measurements, $d \Gamma / d q^{2}$, where $q^{2}=M^{2}\left(e^{+} \nu\right)$ is the square of the four-momentum transfer between initial state $D^{0}$ and final state $K^{-}$or $\pi^{-}$, the hadronic form factors at $q^{2}=0$ times CKM matrix element products $f_{+}^{K}(0)\left|V_{c s}\right|=0.7172 \pm 0.0043$ and $f_{+}^{\pi}(0)\left|V_{c d}\right|=0.1435 \pm 0.0020$ are obtained. Since the precision of current LQCD form factor calculations is not very high, these products are combined with values of $\left|V_{c s}\right|$ and $\left|V_{c d}\right|$ from a SM-constrained fit [54], to extract the hadronic form factors: $f_{+}^{K}(0)=0.7367 \pm 0.0044$ and $f_{+}^{\pi}(0)=0.6395 \pm 0.0090$. Their measured ratio, $f_{+}^{\pi}(0) / f_{+}^{K}(0)=$ $0.868 \pm 0.013$, is in good agreement with a light cone sum rule value $0.84 \pm 0.04$ [59], but with a smaller uncertainty.

The branching fractions of $D^{0} \rightarrow K^{-} \mu^{+} \nu(47100 \pm 259$ observed events $)$ and $\pi^{-} \mu^{+} \nu(2265 \pm 63$ observed events) are measured to be $(3.413 \pm 0.040) \%$ [60] and $(0.272 \pm 0.010) \%$ [61] , respectively, with significantly improved precision compared with previous measurements. With $\left|V_{c s}\right|$ taken from a SM constrained fit [54], $f_{+}^{K}(0)=0.7327 \pm 0.0049$ is obtained, in good agreement with the form factor measured in electronic mode with comparable precision.

For the charged $D$ meson measurements, 1.7 million $D^{+} D^{-}$pairs are tagged, and the absolute decay branching fractions $\mathcal{B}\left(D^{+} \rightarrow \bar{K}^{0} e^{+} \nu\right)=(8.60 \pm 0.16) \%$ and $\mathcal{B}\left(D^{+} \rightarrow \pi^{0} e^{+} \nu\right)=(0.363 \pm$ $0.009) \%$ are determined based on $26008 \pm 168$ and $3402 \pm 70$ observed $D^{+} \rightarrow \bar{K}^{0} e^{+} \nu$ and $\pi^{0} e^{+} \nu$ events, respectively [62]. These results correspond to $f_{+}^{K}(0)=0.725 \pm 0.013$ and $f_{+}^{\pi}(0)=$ $0.622 \pm 0.012$, in agreement with the measurements using neutral $D$ decays with slightly worse precision.

The branching fractions of $D^{+} \rightarrow \bar{K}^{0} \mu^{+} \nu(16516 \pm 130$ observed events $)$ and $\pi^{0} \mu^{+} \nu(1335 \pm 42$ observed events) are measured to be $(8.72 \pm 0.19) \%$ [63] and $(0.350 \pm 0.015) \%$ [61], respectively. The former measurement is ten times more precise than previous results and the latter is a first measurement.

Since the same hadronic form factors occur in $\pi / K e^{+} \nu$ and $\pi / K \mu^{+} \nu$ decays, they cancel in the ratio of branching fractions allowing for a model-independent test of LFU. Tantalizing indications of possible LFU violations have been reported for semileptonic $B$ decays, and these have sparked an interest in more stringent tests in the charm sector, which, if nothing else, could provide useful constraints on models proposed as explanations for the $B$ decay anomalies [64].

The results listed above correspond to branching fraction ratios of $\mathcal{R}_{\pi}^{0} \equiv \frac{\mathcal{B}\left(D^{0} \rightarrow \pi^{-} \mu^{+} \nu\right)}{\mathcal{B}\left(D^{0} \rightarrow \pi^{-} e^{+} \nu\right)}=$ $0.922 \pm 0.037$, and $\mathcal{R}_{\pi}^{+} \equiv \frac{\mathcal{B}\left(D^{+} \rightarrow \pi^{0} \mu^{+} \nu\right)}{\mathcal{B}\left(D^{+} \rightarrow \pi^{0} e^{+} \nu\right)}=0.964 \pm 0.045$ [61]. These are compatible with LFU-based theoretical expectations: $\mathcal{R}_{\pi}=0.985 \pm 0.002$ [65, 66], within 1.7 and 0.5 standard deviations, respectively. Likewise, $\mathcal{R}_{K}^{0} \equiv \frac{\mathcal{B}\left(D^{0} \rightarrow K^{-} \mu^{+} \nu\right)}{\mathcal{B}\left(D^{0} \rightarrow K^{-} e^{+} \nu\right)}=0.974 \pm 0.014$ [60], and $\mathcal{R}_{K}^{+} \equiv \frac{\mathcal{B}\left(D^{+} \rightarrow \bar{K}^{0} \mu^{+} \nu\right)}{\mathcal{B}\left(D^{+} \rightarrow \bar{K}^{0} e^{+} \nu\right)}=1.014 \pm 0.017$, are in agreement with the LFU expected value of $\mathcal{R}_{K}=0.975 \pm 0.001$ [65, 66], within 0.1 and 2.3 standard deviations, respectively. These tests

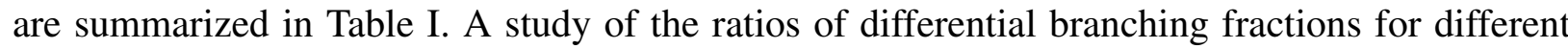
four-momentum transfer regions was also performed [60, 61], and no evidence for LFU violation was found.

Although BESIII also measured many other semi-leptonic decays of $D^{0}, D^{+}$, and $D_{s}^{+}$into final 
TABLE I: Charm decay branching fractions from BESIII experiment, where the first errors are statistical and the second ones systematic.

\begin{tabular}{lccc}
\hline \hline Mode & Number of signals & Branching fraction & Physics implication \\
\hline$D^{+} \rightarrow \mu^{+} \nu$ & $409 \pm 21$ & $(3.71 \pm 0.19 \pm 0.06) \times 10^{-4}$ & $f_{D^{+}}\left|V_{c d}\right|=45.75 \pm 1.20 \pm 0.39 \mathrm{MeV}$ \\
$D_{s}^{+} \rightarrow \mu^{+} \nu$ & $1136 \pm 33$ & $(5.49 \pm 0.16 \pm 0.15) \times 10^{-3}$ & $f_{D_{s}^{+}}\left|V_{c s}\right|=246.2 \pm 3.6 \pm 3.6 \mathrm{MeV}$ \\
\hline$D^{0} \rightarrow K^{-} e^{+} \nu$ & $70727 \pm 278$ & $(3.505 \pm 0.014 \pm 0.033) \%$ & $R_{K}^{0}=0.974 \pm 0.007 \pm 0.012$ \\
$D^{0} \rightarrow K^{-} \mu^{+} \nu$ & $47100 \pm 259$ & $(3.413 \pm 0.019 \pm 0.035) \%$ & \\
\hline$D^{0} \rightarrow \pi^{-} e^{+} \nu$ & $6297 \pm 87$ & $(0.295 \pm 0.004 \pm 0.003) \%$ & \multirow{2}{*}{$R_{\pi}^{0}=0.922 \pm 0.030 \pm 0.022$} \\
$D^{0} \rightarrow \pi^{-} \mu^{+} \nu$ & $2265 \pm 63$ & $(0.272 \pm 0.008 \pm 0.006) \%$ & \\
\hline$D^{+} \rightarrow \bar{K}^{0} e^{+} \nu$ & $26008 \pm 168$ & $(8.60 \pm 0.06 \pm 0.15) \%$ & \multirow{2}{*}{$R_{K}^{+}=1.014 \pm 0.011 \pm 0.013$} \\
$D^{+} \rightarrow \bar{K}^{0} \mu^{+} \nu$ & $16516 \pm 130$ & $(8.72 \pm 0.07 \pm 0.18) \%$ & \\
\hline$D^{+} \rightarrow \pi^{0} e^{+} \nu$ & $3402 \pm 70$ & $(0.363 \pm 0.008 \pm 0.005) \%$ & \multirow{2}{*}{$R_{\pi}^{+}=0.964 \pm 0.037 \pm 0.026$} \\
$D^{+} \rightarrow \pi^{0} \mu^{+} \nu$ & $1335 \pm 42$ & $(0.350 \pm 0.011 \pm 0.010) \%$ & \\
\hline \hline
\end{tabular}

states with a light scalar hadron [67-71], a light vector meson [67, 69-73], and other light pseudoscalar particles [73-75], many of these were first measurements that lack sufficient precision to extract meaningful information on the CKM matrix elements or form factors. For many of these modes, there are still no LQCD form-factor calculations for comparison.

\section{Strong phases in hadronic $D$-meson decays}

Violations of $C P$ invariance in the SM are characterized by the $\alpha, \beta$, and $\gamma$ internal angles of the CKM unitarity triangle (see below) that are determined from various $B$ decay processes [76]. The SM does not specify the values of these angles, but strictly requires that $\alpha+\beta+\gamma=180^{\circ}$ in order to satisfy unitarity. The sum of the current experimental values is $(180.6 \pm 6.8)^{\circ}$, with precision limited by the $\alpha$ and $\gamma$ determinations that are both known to within $\sim \pm 5^{\circ}$ [77]. Improved measurements of these two angles are important.

An improved measurement of the angle $\gamma$ is also needed because it is the only $C P$-violating angle that can be measured in processes that are not mediated by quantum loop diagrams and, thus, not susceptible to the influence of virtual heavy particles that might be associated with new physics. Thus, $\gamma$ is a benchmark reference point for the SM $C P$-violation $(C P V)$ mechanism in searches for non-SM sources of $C P V$, and refined measurements of it are top priorities for the upgraded $\mathrm{LHCb}[5]$ and the Belle II [6] experiments.

Precision determinations of $\gamma$ rely on the measurements of the interference effects between the $B \rightarrow D^{(*)} K^{(*)}$ and $\bar{D}^{(*)} K^{(*)}$ decay amplitudes, and these require as input a precise knowledge of the ( $C P$-conserving) strong-interaction phase differences between the Cabibbo-favoured (transitions between quarks in the same generation which are dominant) and doubly Cabibbo-suppressed (transitions between quarks in different generations which are strongly suppressed) amplitudes for the decays of quantum-correlated $D^{0}-\bar{D}^{0}$ meson systems. BESIII measurements with $D^{0} \bar{D}^{0}$ meson pairs produced at the $\psi(3770)$ resonance peak are uniquely well suited for determining these strong-interaction phase differences [78].

Recent determinations of $\gamma$ from measurements of $B \rightarrow D^{(*)} K^{(*)}$ with $D \rightarrow K_{S}^{0} \pi^{+} \pi^{-}$, which is the most promising channel for future high-precision results, use measured values of the strong- 
interaction phase differences determined by the CLEO-c experiment [79]. The contribution to the final uncertainty from uncertainties of the strong phases' measured values is $\approx \pm 2^{\circ}[80]$. The current BESIII $\psi(3770)$ data set is three and a half times larger than that of CLEO-c and, when fully analyzed, is expected to contribute an uncertainty on $\gamma$ that is of order $1^{\circ}[81]$. This precision should be adequate for the LHCb Run- 2 measurement that has an expected sensitivity of $4^{\circ}$ and the Belle II measurement that will ultimately have an expected precision of $1.5^{\circ}$ [6].

\section{Measurement of absolute $\Lambda_{c}^{+}$decay branching fractions}

Although the $\Lambda_{c}^{+}$baryon was discovered forty years ago [82], its decay properties are still poorly understood. The first model-independent measurement of a $\Lambda_{c}^{+}$decay branching fraction was a 2014 Belle result[83] $\mathcal{B}\left(\Lambda_{c}^{+} \rightarrow p K^{-} \pi^{+}\right)=(6.84 \pm 0.36) \%$. In 2014, BEPCII operated at $\mathrm{E}_{\mathrm{CM}}=4.60 \mathrm{GeV}$, which is $27 \mathrm{MeV}$ above threshold for $e^{+} e^{-} \rightarrow \Lambda_{c}^{+} \bar{\Lambda}_{c}^{-}$production. At this energy, when a $\Lambda_{c}^{\mp}$ is reconstructed, the accompanying $\Lambda_{c}^{ \pm}$is tagged. With a sample of about fourteen thousand tagged $\Lambda_{c}^{+} \bar{\Lambda}_{c}^{-}$events, BESIII reported the first model-independent branching fraction measurements for the semi-leptonic modes $\Lambda_{c}^{+} \rightarrow \Lambda e^{+} \nu$ [84] and $\Lambda \mu^{+} \nu$ [85], and for twelve different hadronic $\Lambda_{c}$ decay modes, including $\mathcal{B}\left(\Lambda_{c}^{+} \rightarrow p K^{-} \pi^{+}\right)=(5.84 \pm 0.35) \%$ [86], which is two standard deviations below the Belle value.

The BESIII $\Lambda_{c}$ measurements were carried out at $\mathrm{E}_{\mathrm{CM}}=4.60 \mathrm{GeV}$, the maximum BEPCII energy, where $\sigma\left(e^{+} e^{-} \rightarrow \Lambda_{c}^{+} \bar{\Lambda}_{c}^{-}\right)=0.24 \pm 0.02 \mathrm{nb}$ [87]. The Belle group reported that this cross-section has a $0.44 \pm 0.18 \mathrm{nb}$ peak at $\mathrm{E}_{\mathrm{CM}}=4.64 \sim 4.66 \mathrm{GeV}$ [88], which is likely a $\Lambda_{c}^{+} \bar{\Lambda}_{c}^{-}$ decay mode of the $Y(4660)$, a charmonium-like $\pi^{+} \pi^{-} \psi(3686)$ resonance found by Belle [89] with mass $4643 \pm 9 \mathrm{MeV} / c^{2}$ [54]. A BEPCII upgrade that is currently underway will increase its maximum energy to $4.9 \mathrm{GeV}$ that will easily cover the $Y(4660)$ peak region, and future BESIII $\Lambda_{c}$ measurements will be done at an energy corresponding to the peak cross-section value with twice the event rate.

\section{DISCOVERY AND STUDY OF CHARMONIUM-LIKE STATES}

All studies of XYZ mesons at $e^{+} e^{-} B$-factories suffer from low event rates and limited statistical precision. In contrast, BESIII can tune the $e^{+} e^{-} \mathrm{CM}$ energy to match the peaks of the vector charmonium-like resonances (the $Y$ mesons), where event rates are high enough to facilitate precise, detailed measurements of their resonance parameters and also search for new states among their decay products.

\section{A. Discovery of $Z_{c}(3900)$ and $Z_{c}(4020)$}

The $Z_{c}(3900)$ hadron was first seen by BESIII as a prominent peak (see Fig. 3 ) in the $\pi^{ \pm} J / \psi$ invariant mass spectrum in a sample of $1.5 \mathrm{~K} e^{+} e^{-} \rightarrow \pi^{+} \pi^{-} J / \psi$ events collected at $\mathrm{E}_{\mathrm{CM}}=$ $4.26 \mathrm{GeV}$, which is near the peak of the $Y(4260)$ resonance [90]. A fit to this signal with a BreitWigner function found its mass and width to be $M=(3899.0 \pm 6.1) \mathrm{MeV} / c^{2}$ and $\Gamma=(46 \pm$ 22) $\mathrm{MeV}$, with a statistical significance greater than 8 standard deviations. The state was named $Z_{c}(3900)$ following the tradition that uses $Z$ to designate a charged quarkonium-like state and a 
subscript $c$ to indicate it contains charm quarks. The $Z_{c}(3900)$ was the first charged charmoniumlike state to be confirmed by other experiments [91, 92] and is a strong candidate for a four-quark meson $\left(Z_{c}^{+}=c \bar{c} u \bar{d} ; Z_{c}^{-}=c \bar{c} d \bar{u}\right)$, where the $c \bar{c}$ pair is needed to account for its decay into a $J / \psi$ charmonium state and the $u$ and $d$ quarks are needed to account for its non-zero electrical charge.
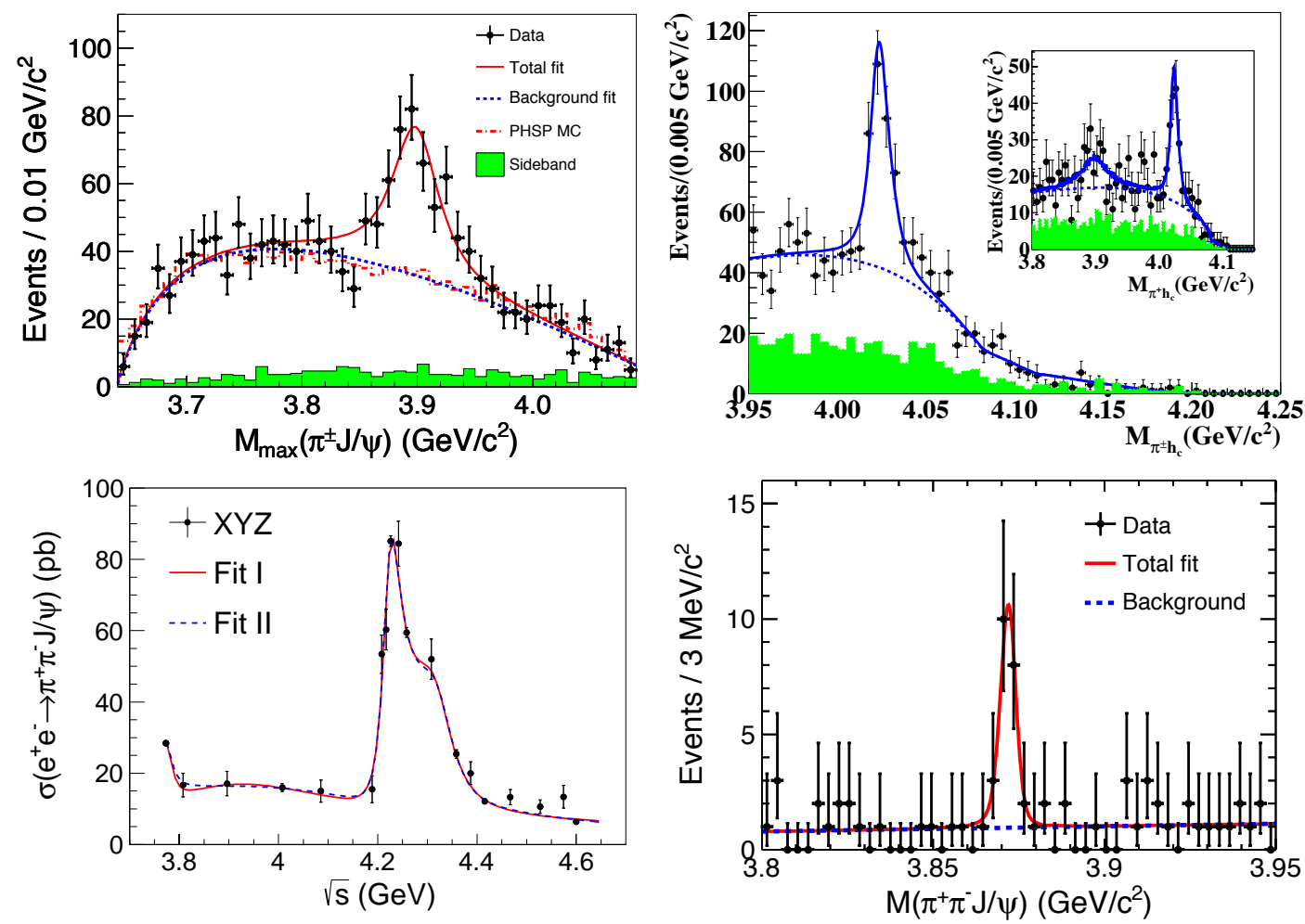

FIG. 3: The $Z_{c}$ (3900) signal observed in $e^{+} e^{-} \rightarrow \pi^{+} \pi^{-} J / \psi$ [90] (top left), the $Z_{c}$ (4020) signal observed in $e^{+} e^{-} \rightarrow \pi^{+} \pi^{-} h_{c}$ [93] (top right), the $Y$ (4260) signal and the fine structure in $e^{+} e^{-} \rightarrow \pi^{+} \pi^{-} J / \psi$ line shape [94] (bottom left), and the $X(3872)$ signal observed in $e^{+} e^{-} \rightarrow \gamma \pi^{+} \pi^{-} J / \psi$ [95] (bottom right). The inset in the top right panel shows the sum of the data at 4.23 and $4.26 \mathrm{GeV}$ with a hint of the $Z_{c}(3900)$ signal.

Subsequently, with $5964 \pm 83 \pi^{+} \pi^{-} J / \psi$ events collected at $\mathrm{E}_{\mathrm{CM}}=4.26$ and $4.23 \mathrm{GeV}$ (where the $e^{+} e^{-} \rightarrow \pi^{+} \pi^{-} J / \psi$ cross-section was found to be larger), a full amplitude analysis of the $\pi^{+} \pi^{-} J / \psi$ system was performed and the spin-parity of the $Z_{c}(3900)$ was determined to be $J^{P}=$ $1^{+}$, and its pole mass and width to be $(3881 \pm 53) \mathrm{MeV} / c^{2}$ and $(52 \pm 36) \mathrm{MeV}$, respectively [96]. The shift in mass and increase in the uncertainties reflect the effects of interference between the $Z_{c}(3900)$ and other amplitudes that were not considered in the initial analysis. The $Z_{c}(3900)$ mass is $5 \mathrm{MeV} / c^{2}$ above the energy threshold for the production of $D \bar{D}^{*}$.

With $e^{+} e^{-} \rightarrow \pi^{ \pm}\left(D \bar{D}^{*}\right)^{\mp}$ events in the same data set, BESIII observed a near-threshold $D \bar{D}^{*}$ mass peak with $J^{P}=1^{+}$that is consistent with $Z_{c}(3900)^{ \pm} \rightarrow\left(D \bar{D}^{*}\right)^{ \pm}$decay [97]. The pole mass and width determined from a fit to the peak are $(3882.2 \pm 1.9) \mathrm{MeV} / c^{2}$ and $(26.5 \pm 2.7) \mathrm{MeV}$, respectively, and in agreement with the values from the $\pi J / \psi$ mode [97, 98]. The measured branching fraction for the $Z_{c}(3900) \rightarrow D \bar{D}^{*}$ decay mode is larger than that for the $\pi J / \psi$ mode by a factor of $6.2 \pm 2.9$. In addition to the well established $\pi J / \psi$ and $D \bar{D}^{*}$ modes, BESIII has reported evidence for $Z_{c}(3900) \rightarrow \rho \eta_{c}$ [99, 100] and $\pi h_{c}$ [93], and an upper limit on $Z_{c}(3900) \rightarrow$ $\pi \psi(3686)$ [101]. 
Since the $Z_{c}(3900)$ mass is near that of the $X(3872)$, a neutral $\pi^{+} \pi^{-} J / \psi$ resonance first observed by Belle in 2003 [102], the two states have been interpreted as isovector and isoscalar $D \bar{D}^{*}$ molecules loosely bound by Yukawa-like nuclear forces [103, 104]. Another possibility is that they are QCD tetraquark states comprising coloured diquarks and diantiquarks tightly bound by the exchange of coloured gluons [105, 106] (The quark and gluon configurations of different kind of hadrons are depicted in Fig. 4p.
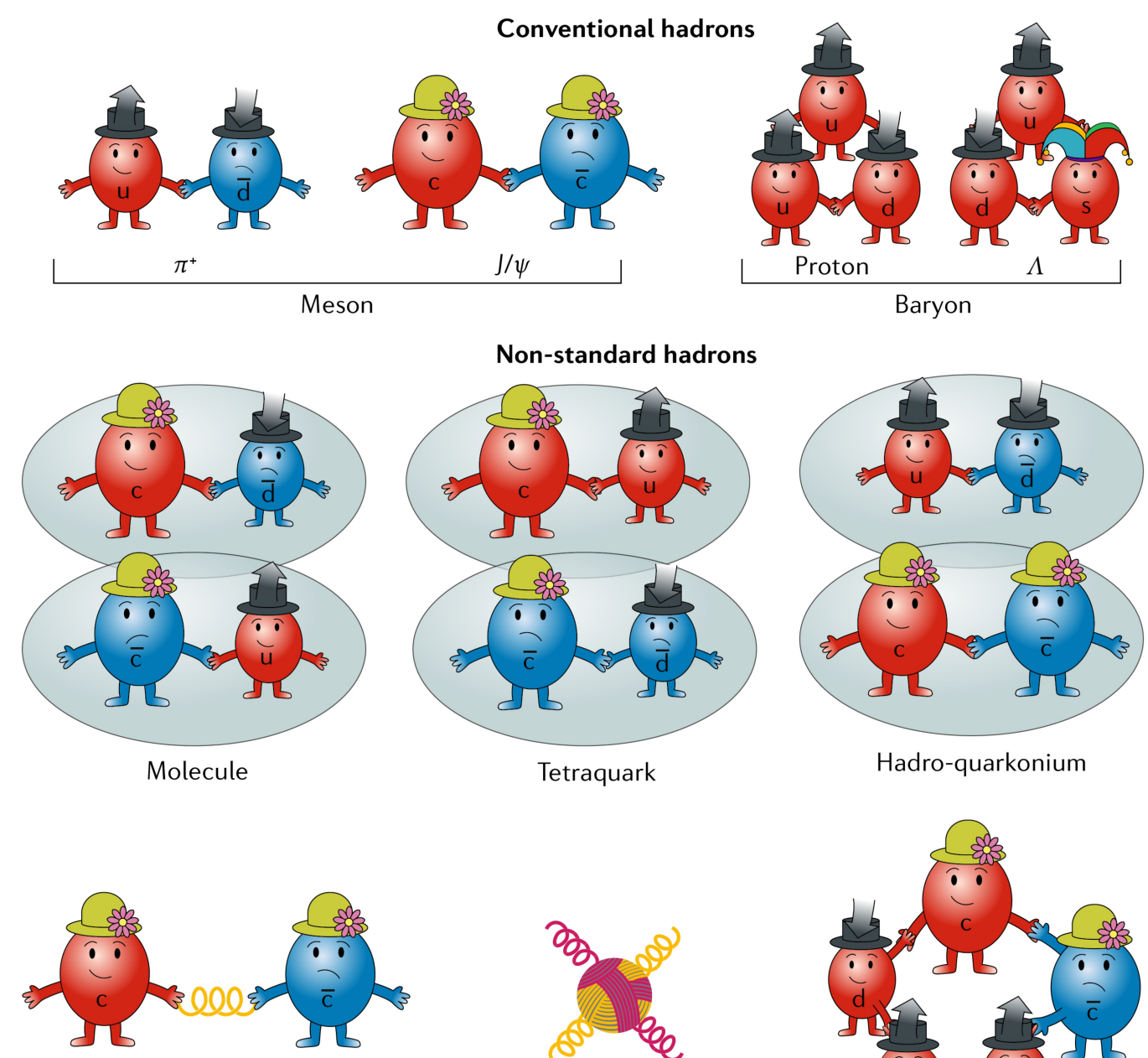

Hybrid

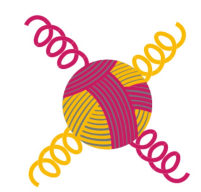

Glueball

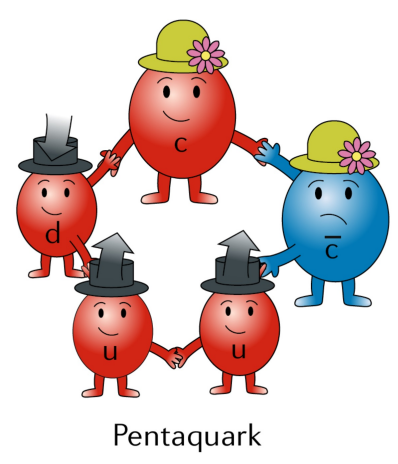

FIG. 4: Conventional and nonstandard hadrons. Conventional mesons are composed of one quark (red characters) and one antiquark (blue characters), conventional baryons are composed of three quarks. The quarks can have different flavours such as up (u), down (d), charm (c) or strange (s). Hadrons with other configurations are referred to as nonstandard. Nonstandard hadrons include hadron-hadron molecules, diquarkdiantiquark tetraquark mesons, hadro-quarkonia, quark-antiquark-gluon hybrids, multi-gluon glueballs and pentaquark baryons.

Many of the models that were proposed for the $Z_{c}(3900)$ predict the existence of a similar state near the $D^{*} \bar{D}^{*}$ threshold. Although there are no strong indications of a state near the $D^{*} \bar{D}^{*}$ mass 
threshold in BESIII's $e^{+} e^{-} \rightarrow \pi^{+} \pi^{-} J / \psi$ or $\pi D \bar{D}^{*}$ data samples, distinct signals for the predicted state, the $Z_{c}(4020)$, were discovered in the $e^{+} e^{-} \rightarrow \pi^{+} \pi^{-} h_{c}$ and $\pi D^{*} \bar{D}^{*}$ channels.

An analysis of $e^{+} e^{-} \rightarrow \pi^{+} \pi^{-} h_{c}$ events collected with $\mathrm{E}_{\mathrm{CM}}$ at and near the $Y(4260)$ resonance peak found distinct evidence for a nonstandard charmonium-like structure in the $\pi^{ \pm} h_{c}$ invariant mass distribution as shown in Fig. 3 [93]. The mass and width of this structure were determined to be $(4022.9 \pm 2.8) \mathrm{MeV} / c^{2}$ and $(7.9 \pm 3.7) \mathrm{MeV}$, respectively, with a statistical significance greater than 8.9 standard deviations. This discovery was only possible because of the very clean experimental environment of $e^{+} e^{-}$collisions in the $\tau$-charm threshold energy region uniquely facilitates the isolation of distinct $h_{c}$ meson signals. Neither the BaBar and Belle $B$-factory nor the $\mathrm{LHCb}$ experiment has ever seen an $h_{c}$ signal.

BESIII also observed a strong $Z_{c}(4020)^{ \pm} \rightarrow\left(D^{*} \bar{D}^{*}\right)^{ \pm}$signal in $e^{+} e^{-} \rightarrow\left(D^{*} \bar{D}^{*}\right)^{ \pm} \pi^{\mp}$ events at $\mathrm{E}_{\mathrm{CM}}=4.26 \mathrm{GeV}$. The measured mass and width in this channel are $(4026.3 \pm 4.5) \mathrm{MeV} / \mathrm{c}^{2}$ and $(24.8 \pm 9.5) \mathrm{MeV}$, respectively, and the statistical significance is 13 standard deviations [107].

The $J^{P}=1^{+} Z_{c}$ states found by BESIII bear an uncanny resemblance to analogous $Z_{b}$ charged bottomonium-like $1^{+}$states discovered by Belle [30] in 2011. The $Z_{c}(3900)$ and $Z_{c}(4020)$ are $\sim 5 \mathrm{MeV} / c^{2}$ above the $D \bar{D}^{*}$ and $D^{*} \bar{D}^{*}$ thresholds, respectively, whereas the $Z_{b}(10610)$ and $Z_{b}(10650)$ are $\sim 3 \mathrm{MeV} / c^{2}$ above the respective $B \bar{B}^{*}$ and $B^{*} \bar{B}^{*}$ thresholds (where $B$ denotes the $B$ meson), and they all have similar widths. These features are similar to those expected for kinematic effects that can produce peaks just above thresholds that look like resonances, but have nothing to do with bona fide mesons [108-110]. This possibility was studied for the specific case of the $Z_{c}(3900)$ in Ref. [111], which concluded that the characteristics of the narrow $Z_{c}(3900) \rightarrow D \bar{D}^{*}$ signal [97] establishes the presence of a genuine meson-like pole in the $D \bar{D}^{*}$ $S$-matrix. A more general discussion of this issue is provided in Ref. [112].

In the QCD tetraquark and molecular pictures, the $Z_{c}(3900)^{ \pm}$and $Z_{c}(4020)^{ \pm}$states are the $I_{3}= \pm 1$ members of isospin triplets. BESIII confirmed this by observing their neutral, isospin $I_{3}=0$ partners: the $Z_{c}(3900)^{0}$, in both the $\pi^{0} J / \psi[113]$ and $\left(D \bar{D}^{*}\right)^{0}$ [114] decay modes; and the $Z_{c}(4020)^{0}$, in the $\pi^{0} h_{c}$ [115] and $\left(D^{*} \bar{D}^{*}\right)^{0}$ [116] decay modes. These observations establish the $Z_{c}(3900)$ and $Z_{c}(4020)$ as isovector states with even $G$-parity.

\section{B. Improved understanding of the $Y(4260)$}

The $Y$ charmonium-like states are vector mesons with spin-parity quantum numbers $J^{P C}=$ $1^{--}$, the same quantum numbers as the photon. As a result, they can be produced directly in $e^{+} e^{-}$annihiations via a single virtual photon, that is $e^{+} e^{-} \rightarrow Y$. These states, which include the $Y(4260)$ [117], the $Y(4360)$ [89, 118], and the $Y(4660)$ [89], have strong couplings to $c \bar{c}$ charmonium final states, in contrast to conventional vector $c \bar{c}$ charmonium states in the same energy region (such as $\psi(4040), \psi(4160)$, and $\psi(4415)$ ) that dominantly couple to pairs of $D$ mesons [54]. These $Y$ states are candidates for a variety of types of nonstandard hadrons including: molecules [104, 119,-121]; diquark-diantiquarks [122, 123]; QCD-hybrids [124, 125]; and hadrocharmonia [126] (See Fig. 4).

A series of high-luminosity data sets taken with $\mathrm{E}_{\mathrm{CM}}$ spanning the $Y(4260)$ mass region provided more precise measurements of the $\mathrm{E}_{\mathrm{CM}^{-}}$-dependence of the cross-section $\sigma\left(e^{+} e^{-} \rightarrow\right.$ $\left.Y(4260) \rightarrow \pi^{+} \pi^{-} J / \psi\right)$ [94] than any of the seven previous measurements that were based on samples of ISR events in higher energy $e^{+} e^{-}$collision data [117, 127]. With an order-of-magnitude better statistical precision, the BESIII measurements revealed a previously unnoticed composite 
structure in the $Y(4260)$ resonance line-shape, evident in Fig. 3, that can be described by the overlap of a narrow peak with mass $(4222.0 \pm 3.4) \mathrm{MeV} / \mathrm{c}^{2}$ and width $(44.1 \pm 4.7) \mathrm{MeV}$, and a wider one with mass $(4320 \pm 13) \mathrm{MeV} / \mathrm{c}^{2}$ and width $(101 \pm 27) \mathrm{MeV}$. Compared to the previous 2016 world average values for the $Y(4260)$ [128], the mass of $4222 \mathrm{MeV} / c^{2}$ resonance is about $30 \mathrm{MeV} / \mathrm{c}^{2}$ lower and its width is nearly a factor of 3 narrower.

BESIII also observed additional decay modes of the lower-mass $Y(4260)$ peak, including: $Y(4260) \rightarrow \pi^{+} \pi^{-} h_{c}$ [129]; $\omega \chi_{c 0}[130] ; \pi^{+} \pi^{-} \psi(3686)$ [101]; and the $\pi D^{*}[131]$ open-charmmeson mode. Curiously, the $4320 \mathrm{MeV} / \mathrm{c}^{2}$ peak is not seen in any of these additional channels. The resonant parameters of the $Y(4260)$ measured in different modes are listed in Table II.

TABLE II: Resonant parameters of the $Y(4260)$ from different modes measured at BESIII. The cross sections measured at CM energy $4.226 \mathrm{GeV}$ are also listed.

\begin{tabular}{cccc}
\hline \hline Mode & Mass $\left(\mathrm{GeV} / c^{2}\right)$ & Width $(\mathrm{MeV})$ & $\sigma$ at $\sqrt{s}=4.226 \mathrm{GeV}(\mathrm{pb})$ \\
\hline$e^{+} e^{-} \rightarrow \pi^{+} \pi^{-} J / \psi$ & $4222.0 \pm 3.1 \pm 1.4$ & $44.1 \pm 4.3 \pm 2.0$ & $85.1 \pm 1.5 \pm 4.9$ \\
$e^{+} e^{-} \rightarrow \pi^{+} \pi^{-} h_{c}$ & $4218.4_{-4.5}^{+5.5} \pm 0.9$ & $66.0_{-8.3}^{+12.3} \pm 0.4$ & $55.2 \pm 2.6 \pm 8.9$ \\
$e^{+} e^{-} \rightarrow \omega \chi_{c 0}$ & $4218.5 \pm 1.6 \pm 4.0$ & $28.2 \pm 3.9 \pm 1.6$ & $55.4 \pm 6.0 \pm 5.9$ \\
$e^{+} e^{-} \rightarrow \pi^{+} \pi^{-} \psi(3686)$ & $4209.5 \pm 7.4 \pm 1.4$ & $80.1 \pm 24.6 \pm 2.9$ & $21.3 \pm 1.1 \pm 1.6$ \\
$e^{+} e^{-} \rightarrow \pi^{+} D^{0} D^{*-}+$ c.c. & $4228.6 \pm 4.1 \pm 6.3$ & $77.0 \pm 6.8 \pm 6.3$ & $252 \pm 5 \pm 15$ \\
\hline \hline
\end{tabular}

The $Y(4260)$ has attracted a considerable amount of attention ever since its discovery in 2005 . The presence of nearby thresholds for $D_{s}^{*+} D_{s}^{*-}, D \bar{D}_{1}(2420)$, and $\omega \chi_{c J}$ production, and its mass overlap with the $\psi(4160)$ and $\psi(4415)$ conventional vector $c \bar{c}$ charmonium states complicate its interpretation. Whereas BESIII will continue to supply more results on the $Y(4260)$ properties, sophisticated theoretical treatments are likely needed to understand the nature of this state.

\section{Commonality between the $X(3872), Y(4260)$, and $Z_{c}(3900)$}

With data taken with $\mathrm{E}_{\mathrm{CM}}$ at and near the $Y(4260)$ resonance peak, BESIII discovered a clear signal for $X(3872)$ production in association with a $\gamma$-ray [95], as shown in Fig. 3. The $\mathrm{E}_{\mathrm{CM}^{-}}$ dependence of the $e^{+} e^{-} \rightarrow \gamma X(3872)$ cross-section is suggestive of a $Y(4260) \rightarrow \gamma X(3872)$ decay process, which indicates that there might be some common features to the internal structures of the $Z_{c}(3900), Y(4260)$, and $X(3872)$.

All the final state particles in $e^{+} e^{-} \rightarrow \gamma X(3872)$ followed by $X(3872)$ decays are recorded in the detector and there is no other particles in an event. This makes it well suited for studies of $X(3872)$ decays to final states that include $\gamma$ rays and $\pi^{0}$ mesons. BESIII exploited this to make the first observation of the $X(3872) \rightarrow \pi^{0} \chi_{c 1}$ decay mode [132], a process that would be strongly suppressed for a $c \bar{c}$ state, but allowed for a four-quark state [133]. This process was also used to determine the branching fraction for $X(3872) \rightarrow \omega J / \psi$ with a two-fold improvement in precision over previous measurements [134]. 


\section{STUDY OF LIGHT HADRONS IN $J / \psi$ DECAYS}

\section{A. Scalar and tensor glueball searches with partial wave analyses}

An intriguing QCD prediction that is yet to be experimentally confirmed is the existence of mesons comprised only of gluons, with no quark content, and commonly referred to as glueballs [135]. Glueballs are electrically neutral, zero isospin SU(3) singlets [136]. Two gluons form scalar $\left(0^{++}\right)$, pseudoscalar $\left(0^{-+}\right)$, tensor $\left(2^{++}\right)$glueballs and so on. Radiative $J / \psi$ decays proceed via the $J / \psi \rightarrow \gamma g g$ process and are expected to be prolific sources of glueballs with mass below $3 \mathrm{GeV} / c^{2}$. BESIII has performed systematic studies of radiative $J / \psi$ decays using partial wave analysis (PWA) techniques to search for and characterize glueball candidates.

The lowest mass scalar glueball is expected to have a mass between 1 and $2 \mathrm{GeV} / c^{2}$ and decay into meson-antimeson pairs [137]. BESIII found three scalar mesons in this mass range in the radiative decay processes $J / \psi \rightarrow \gamma \pi^{0} \pi^{0}$ [138], $\gamma K_{S}^{0} K_{S}^{0}$ [139], and $\gamma \eta \eta$, where $\eta$ stands for the eta meson [140]: the $f_{0}(1370), f_{0}(1500)$, and $f_{0}(1710)$. Two conventional $q \bar{q}$ scalar mesons are needed to account for the $S U(3)$ octet and singlet members of the $1^{3} P_{0}$ meson nonet, leaving one of them as a candidate for a scalar glueball. One interpretation is that these three mesons mix and, thus, are the three orthogonal mixtures of $n \bar{n}=(u \bar{u}+d \bar{d}) / \sqrt{2}, s \bar{s}$, and $\mathcal{G}$, that are the two $S U(3)$ $q \bar{q}$ states and a glueball. Of the three candidates, the $f_{0}(1710)$ has the highest production rate; the branching fraction for $J / \psi \rightarrow \gamma f_{0}(1710)$ is nearly ten times higher than those for $f_{0}(1370)$ and $f_{0}(1500)$, and compatible with an LQCD calculated value for a glueball [141]. This suggests that the $f_{0}(1710)$ has the largest gluon component. Better measurements of the couplings of these states to $\pi^{+} \pi^{-}, K \bar{K}, \eta \eta$, and $\eta \eta^{\prime}$ will supply additional insight into the relative $n \bar{n}, s \bar{s}$, and $\mathcal{G}$ content of these states.

The lowest lying tensor glueball is expected to have a mass above $2 \mathrm{GeV} / c^{2}$ [137], and PWA of $J / \psi \rightarrow \gamma K_{S}^{0} K_{S}^{0}$ [139], $\gamma \eta \eta$ [140], and $\gamma \phi \phi$ [142] revealed a tensor meson, the $f_{2}(2340)$, that decays to each one of these channels. However, its coupling to the $\pi \pi$ mode has not been established [138] and its total production rate appears to be substantially lower than the LQCD calculated value [143], which is of order $1 \%$. This may be because there are a number of $f_{2}(2340)$ decay modes that have not yet been identified, which is reasonable for a state with such high mass. Thus, much more effort is needed to establish and characterize the lowest tensor glueball.

A pseudoscalar $\left(0^{-+}\right)$glueball could also be produced in radiative $J / \psi$ decays, but for these the dominant decay modes would be to three pseudoscalars such as $\eta^{(\prime)} \pi \pi, \eta^{(\prime)} K \bar{K}$, and $K \bar{K} \pi$. As noted below, the identification of a pseudoscalar glueball is not easy.

\section{B. Study of states close to the $p \bar{p}$ mass threshold}

A distinct, narrow peak just at the $p \bar{p}$ threshold was observed in $J / \psi \rightarrow \gamma p \bar{p}$ by BESII, the second phase of the BES project, in a data set containing 58 million $J / \psi$ decays [144]. This peak was subsequently confirmed and its spin-parity was measured to be $0^{-+}$by BESIII with 225 million $J / \psi$ events [145]. BESII also observed an $\eta^{\prime} \pi^{+} \pi^{-}$invariant mass peak in $J / \psi \rightarrow$ $\gamma \eta^{\prime} \pi^{+} \pi^{-}$decays, the $X(1835)$ [146], that was suggested as being due to an $\eta^{\prime} \pi^{+} \pi^{-}$decay mode of the same state as that seen in $p \bar{p}$ [147]. Subsequent BESIII studies of the $X(1835) \rightarrow \eta^{\prime} \pi^{+} \pi^{-}$ line shape with a 1.3 billion $J / \psi$ event sample revealed an anomalous structure in its line shape, 
that is centered at the $p \bar{p}$ threshold (see Fig. 5), which could be equally well described as the interference with a new narrow resonance that has a mass nearly equal to $2 m_{p}$ or a wide resonance with an anomalously strong coupling to the $p \bar{p}$ final state [148].

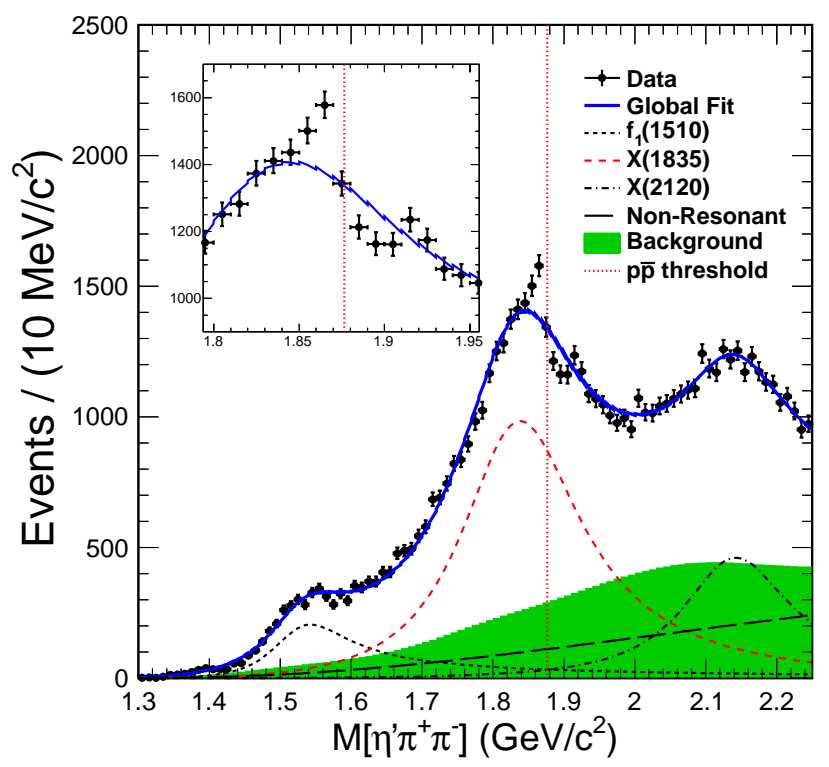

FIG. 5: The $\eta^{\prime} \pi^{+} \pi^{-}$invariant mass spectra in $J / \psi \rightarrow \gamma \eta^{\prime} \pi^{+} \pi^{-}$events [148]. The dotted vertical line shows the position of $p \bar{p}$ mass threshold; the blue curve shows the results for a fit that ignores the influence of the $p \bar{p}$ threshold. The inset shows the detail around the $p \bar{p}$ threshold.

States in this mass region were also reported in $J / \psi \rightarrow \omega \eta \pi^{+} \pi^{-}$[149] and $\gamma 3\left(\pi^{+} \pi^{-}\right)$[150], and these also have line shape distortions near the $p \bar{p}$ mass threshold. However, no near-threshold $p \bar{p}$ structures are observed in $J / \psi$ decays to $\omega p \bar{p}$ [151] or $\phi p \bar{p}$ [152].

The difficulty in drawing solid conclusions about the states in this mass region is that the extracted resonance parameters are strongly model-dependent. They depend on the parameterization of the resonant amplitudes, the way different amplitudes interfere with each other, and how they are affected by the opening of nucleon-antinucleon decay channels at the $2 m_{N}$ thresholds, where $m_{N}$ is the nucleon mass. As a result, the inferred masses and widths of these states can vary over a wide range of values, with correspondingly large uncertainties. In addition, ambiguities inherent to the effects of interference preclude precise production rate measurements.

Very elaborate, coupled-channel PWA of the different processes may be the key for extracting reliable information from the data and for understanding the nature of meson states in this mass region [153]. This is an important future direction for BESIII studies of light meson spectroscopy.

\section{First observation of $a_{0}^{0}(980) \leftrightarrow f_{0}(980)$ mixing}

Five decades have passed since the discovery [154, 155] of the $a_{0}^{0, \pm}(980)$ and $f_{0}(980)$ scalar mesons (with $J^{P C}=0^{++}$), but their nature remains controversial. These two nearly-equal mass states, with different isospin and decay modes, have defied attempts to classify them as conventional $q \bar{q}$ mesons [156]. They have long been considered candidates for $K \bar{K}$ molecules [157], 
QCD tetraquarks [158-162], or QCD hybrids [163]. In 1979, measurements of $a_{0}-f_{0}$ mixing were proposed as sensitive probes into the nature of these states [164].

Forty years later, BESIII made the first experimental observations of this process [165]. Using 1.3 billion $J / \psi$ and 0.45 billion $\psi(3686)$ events, BESIII detected distinct signals for $J / \psi \rightarrow$ $\phi f_{0}(980)$ events in which the $f_{0}$ mixes into an $a_{0}^{0}(980)$ that decays into $\eta \pi^{0}$, and $\chi_{c 1} \rightarrow \pi^{0} a_{0}^{0}(980)$ events where the $a_{0}^{0}$ mixes into an $f_{0}(980)$ that decays to $\pi^{+} \pi^{-}$. The statistical significances are 7.2 and 5.5 standard deviations, respectively. The extracted mixing intensities favour the QCD tetraquark scenario.

\section{BARYON POLARIZATION IN $e^{+} e^{-}$ANNIHILATION AND $J / \psi$ DECAYS}

Baryons produced directly via $e^{+} e^{-}$annihilation into baryon-antibaryon $(B \bar{B})$ pairs or $J / \psi \rightarrow$ $B \bar{B}$ decays can be polarized transversely due to a non-zero relative phase $\Delta \Phi$ between the two complex amplitudes that govern this process [166, 167]. This polarization was generally expected to be small and, thus, it was big surprise when BESIII discovered that, in fact, the polarization of $\Lambda$ (and $\bar{\Lambda}$ ) hyperons produced in $J / \psi \rightarrow \Lambda \bar{\Lambda}$ decays is substantial [168]. A non-zero polarization $\vec{P}_{\Lambda}$ enables separate measurements of $\alpha_{-}$and $\alpha_{+}$, the parity-violating parameters that characterize the final state $\pi^{-}$and $\pi^{+}$angular distributions for polarized $\Lambda \rightarrow p \pi^{-}$and $\bar{\Lambda} \rightarrow \bar{p} \pi^{+}$decays: $d n_{ \pm} / d \cos \theta_{\pi^{ \pm}} \propto 1-\alpha_{ \pm}\left|\vec{P}_{\Lambda}\right| \cos \theta_{\pi^{ \pm}}$, where $\theta_{\pi^{ \pm}}$is the $\pi^{ \pm}$direction relative to $\vec{P}_{\Lambda}$. Prior to the BESIII discovery, it was thought that testing $C P$ with $J / \psi \rightarrow \Lambda \bar{\Lambda}$ pairs produced via $e^{+} e^{-}$ collisions would require a polarized $e^{-}$beam.

With 1.3 billion $J / \psi$ events collected in 2009 and 2012, a total of 420593 fully reconstructed $J / \psi \rightarrow \Lambda \bar{\Lambda}$ events with $\Lambda \rightarrow p \pi^{-}$and $\bar{\Lambda} \rightarrow \bar{p} \pi^{+}$were isolated with a nearly negligible, 399-event background. The data is described well with a large relative phase $\Delta \Phi=(42.4 \pm 0.8)^{\circ}$ as shown in Fig. 6, whereas $\Delta \Phi=0$ is clearly excluded by the data. The transverse polarization of the $\Lambda$ $(\bar{\Lambda})$, which depends on $\cos \theta_{\Lambda}$, the $\Lambda$ direction relative to the $e^{+} e^{-}$beam axis, is shown in Fig. 6 , the values range between $-25 \%$ and $+25 \%$.

For $\Lambda \rightarrow p \pi^{-}$, BESIII measured $\alpha_{-}=0.750 \pm 0.010$, which is more than five standard deviations higher than the previous world average value of $\alpha_{-}=0.642 \pm 0.013$ that was based entirely on pre-1974 measurements [171-175]. The measured $\bar{\Lambda} \rightarrow \bar{p} \pi^{+}$asymmetry parameter, $\alpha_{+}=-0.758 \pm 0.012$, is also high, and consistent, within uncertainties, with the $\Lambda$ result. The $C P$-violating asymmetry, $A_{\mathrm{CP}} \equiv\left(\alpha_{-}+\alpha_{+}\right) /\left(\alpha_{-}-\alpha_{+}\right)=-0.006 \pm 0.014$, is compatible with zero and a factor of two more sensitive than the best previous $\Lambda$-based measurement, $A_{\mathrm{CP}}=$ $+0.013 \pm 0.022$ [176]. This is still well above the SM expectation of $A_{\mathrm{CP}}^{\mathrm{SM}} \sim 10^{-4}$ based on the CKM mechanism [177].

BESIII used $47009 \mathrm{~J} / \psi \rightarrow \Lambda\left(p \pi^{-}\right) \bar{\Lambda}\left(\bar{n} \pi^{0}\right)$ signal events with a negligible 66-event background to measure $\bar{\alpha}_{0}$, the parity violating parameter for $\bar{\Lambda} \rightarrow \bar{n} \pi^{0}$. The result, $\bar{\alpha}_{0}=$ $-0.692 \pm 0.017$, is a threefold improvement on the only previous measurement [178]; the 3 standard deviations difference from $\alpha_{+}$could reflect the presence of an isospin=3/2 component of the $\pi \bar{N}$ final state.

The $A_{\mathrm{CP}}$ measurement is based on a ratio of asymmetries in which many detector-related systematic effects cancel, and the current quoted precision is limited by statistical uncertainties. BESIII's accumulated $J / \psi$ data set has recently grown to 10 billion events, and this should provide a threefold reduction in the $A_{\mathrm{CP}}$ uncertainty. In addition, BESIII is extending similar analyses 

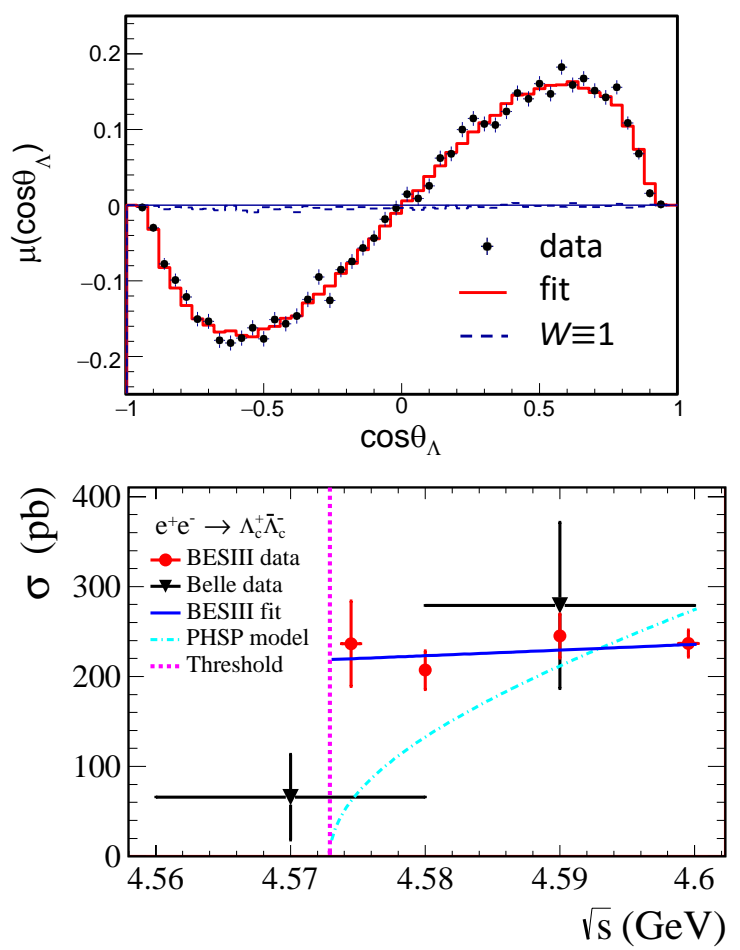
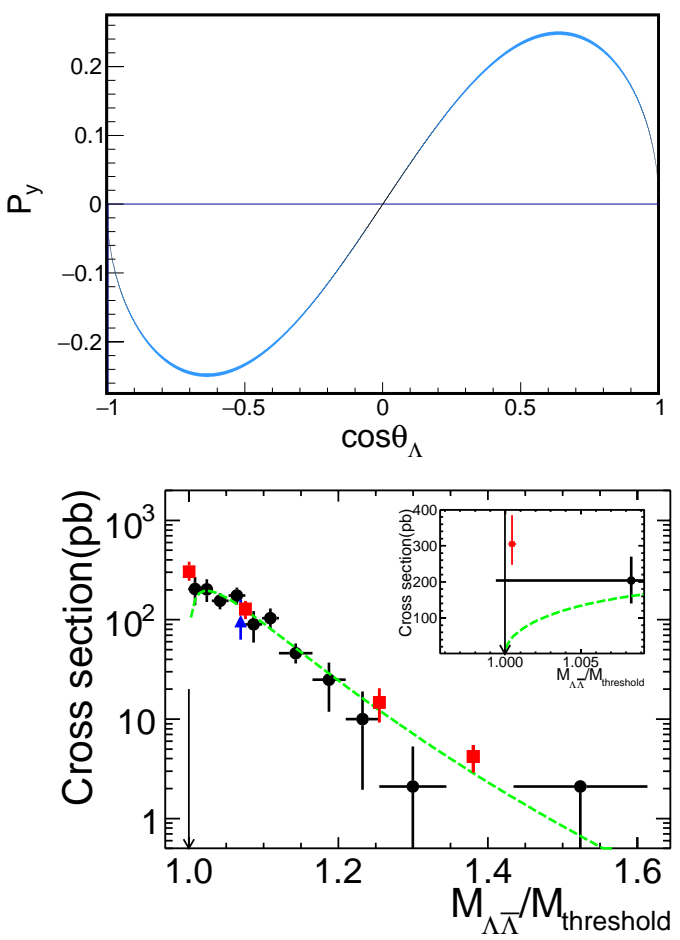

FIG. 6: BESIII measurements on baryon properties. (Top left) The fitted results and (top right) the transverse polarization $P_{y}$ as a function of $\cos \theta_{\Lambda}$ for $J / \psi \rightarrow \Lambda \bar{\Lambda} \rightarrow p \pi^{-} \bar{p} \pi^{+}$events [168]. The dashed histogram in the top left panel shows the no polarization scenario. (Bottom left) The near-threshold $e^{+} e^{-} \rightarrow \Lambda_{c}^{+} \bar{\Lambda}_{c}^{-}$ cross sections measured by BESIII (red points) and Belle (black points) experiments and comparison with phase space model (dash-dot cyan curve) [87]. The vertical dashed line indicates the threshold. (Bottom right) The near-threshold $e^{+} e^{-} \rightarrow \Lambda \bar{\Lambda}$ cross section measured by BESIII (red squares), BaBar (black dots), and DM2 (blue triangles) [169]. The inset is an expanded view on a linear scale near the threshold. The green dashed lines are a phenomenological fit result based on a perturbative quantum chromodynamics prediction [170], the black arrows indicate the threshold.

to $J / \psi \rightarrow \Xi \bar{\Xi}$ and $\psi(3686) \rightarrow \Omega^{-} \bar{\Omega}^{+}$hyperon pairs. For these, the decay final states include a $\Lambda(\bar{\Lambda})$ whose polarization can be determined from its decay asymmetry. This feature provides the capability of additional $C P$ tests [179] that are potentially more sensitive to new physics than $A_{\mathrm{CP}}$.

The CKM mechanism for $C P V$ in the $\mathrm{SM}$ fails to explain the matter-antimatter asymmetry of the Universe by more than 10 orders-of-magnitude [180]. This suggests that additional, heretofore undiscovered, $C P$ violating processes occur, which has motivated intensive searches for new sources of $C P V$ in bottom-quark decays [6, 181-183] and neutrino oscillations [184, 185]. BESIII's capability to test $C P$ symmetry in the decays of polarized, quantum-entangled hyperon pairs produced via $J / \psi \rightarrow B \bar{B}$ adds an exciting new dimension to the study of $C P$ violations.

Searches for new sources of $C P V$ have been elevated to a new level of interest by the recent LHCb discovery of $C P V$ in $D^{0} \rightarrow K^{+} K^{-}$and $D^{0} \rightarrow \pi^{+} \pi^{-}$decays. They measure the time- 
integrated $C P V$ asymmetry

$$
\begin{aligned}
\Delta A_{\mathrm{CP}} & =\frac{\Gamma\left(D \rightarrow K^{+} K^{-}\right)-\Gamma\left(\bar{D} \rightarrow K^{+} K^{-}\right)}{\Gamma\left(D \rightarrow K^{+} K^{-}\right)+\Gamma\left(\bar{D} \rightarrow K^{+} K^{-}\right)}-\frac{\Gamma\left(D \rightarrow \pi^{+} \pi^{-}\right)-\Gamma\left(\bar{D} \rightarrow \pi^{+} \pi^{-}\right)}{\Gamma\left(D \rightarrow \pi^{+} \pi^{-}\right)+\Gamma\left(\bar{D} \rightarrow \pi^{+} \pi^{-}\right)} \\
& =(-0.154 \pm 0.029) \%
\end{aligned}
$$

where $D(\bar{D})$ is a $D^{0}\left(\bar{D}^{0}\right)$ at time $t=0$ [186]. The significance of the $\Delta a_{\mathrm{CP}}$ deviation from zero is 5.3 standard deviations, making this the first observation of $C P V$ in the charm-quark sector. The measured $\Delta A_{\mathrm{CP}}$ value is at the high end of theoretical estimates for its SM value, which range from $10^{-4}$ [187] to $10^{-3}$ [188-191]. The LHCb result is intriguing, because it may be a sign of the long-sought-for non-SM mechanism for $C P V$. However, uncertainties in the calculations of the SM expectation for $\Delta a_{\mathrm{CP}}$ make it impossible to either establish or rule out this possibility. However, BESIII's current $\sim 10^{-2}$ level of sensitivity on $A_{\mathrm{CP}}$ is still two orders-ofmagnitude above the highest conceivable SM effects [177]. Any non-zero measurement of $A_{\mathrm{CP}}$ in the intervening range would be an unambiguous signature for new physics.

\section{MEASUREMENTS OF TIME-LIKE BARYON ELECTROMAGNETIC FORM FACTORS}

The electric charge and magnetization distributions inside baryons are described by electric and magnetic form factors (FFs), $G_{E}\left(q^{2}\right)$ and $G_{M}\left(q^{2}\right)$, where $q^{2}$ is the square of the four-momentum transferred in the process. Historically, huge efforts have been made to determine space-like $\left(q^{2}<\right.$ $0)$ nucleon FFs by electron-nucleon elastic scattering experiments. In contrast, time-like FFs $\left(q^{2}>\right.$ $4 m_{B}^{2}$ ), which are measured in $e^{+} e^{-} \rightarrow B \bar{B}$ or $p \bar{p} \rightarrow e^{+} e^{-}$annihilation reactions, with $q^{2}=\mathrm{E}_{\mathrm{CM}}^{2}$, are less well studied [170].

The behaviour of the time-like FFs near the $B \bar{B}$ mass threshold is particularly interesting. In $e^{+} e^{-} \rightarrow B \bar{B}$, the baryon pair is produced in a short-distance interaction mediated by a hard, $q^{2} \geq 4 m_{B}^{2}$, virtual photon. However, the final state $B$ and $\bar{B}$ are nearly at rest relative to each other in a non-relativistic, long-distance system. In the case where $B$ and $\bar{B}$ are electrically charged, their final state interactions are modified by their mutual Coulomb force. For a point-like charged particle, this Coulomb interaction produces an abrupt jump in the production cross-section right at threshold of $\Delta \sigma=\pi^{2} \alpha^{3} / 2 m_{B}^{2}$; for $m_{B}=m_{p}, \Delta \sigma=0.85 \mathrm{nb}$ [192, 193]. Amazingly, BaBar [194] and CMD-3 [195] measurements of $e^{+} e^{-} \rightarrow p \bar{p}$ have seen an abrupt jump in the $e^{+} e^{-} \rightarrow p \bar{p}$ cross-section at the $\mathrm{E}_{\mathrm{CM}}=2 m_{p}$ threshold that is consistent with the $0.85 \mathrm{nb}$ expectation for a point-like charged particle. BESIII measurements of $\sigma\left(e^{+} e^{-} \rightarrow \Lambda_{c}^{+} \bar{\Lambda}_{c}^{-}\right)$, shown in Fig. 6, have a $\Delta \sigma=0.236 \pm 0.047 \mathrm{nb}$ threshold jump [87] that is larger, although marginally consistent with the $\Delta \sigma=0.14 \mathrm{nb}$ expectation for an $m_{B}=m_{\Lambda_{c}^{+}}$point-like charged particle.

In the case of neutral particles, there is no Coulomb interaction and no mechanism for an abrupt jump at the threshold; the cross-section is expected to grow as the available phase space, which is proportional to $\beta_{f}$, the relative velocity of the two final-state particles. However, in another big surprise, $e^{+} e^{-} \rightarrow n \bar{n}$ measurements did not confirm a $\sigma \propto \beta_{n}$ behaviour, instead they also showed an abrupt threshold jump [196, 197] that is consistent (within large uncertainties) with the $0.85 \mathrm{nb}$ jump seen for $e^{+} e^{-} \rightarrow p \bar{p}$.

Early measurements of $e^{+} e^{-} \rightarrow \Lambda \bar{\Lambda}$ showed some evidence for a non-zero, near-threshold cross-section, but these had large uncertainties and were confined to $\mathrm{E}_{\mathrm{CM}}$ values corresponding to $\beta_{\Lambda} \geq 0.3$ [198, 199]. In 2017, BESIII reported measurements of $e^{+} e^{-} \rightarrow \Lambda \bar{\Lambda}$ for energies 
ranging from $\mathrm{E}_{\mathrm{CM}}=2.2324 \mathrm{GeV}\left(1 \mathrm{MeV}\right.$ above the $2 m_{\Lambda}$ threshold with $\left.\beta_{\Lambda} \approx 0.03\right)$ to $\mathrm{E}_{\mathrm{CM}}=$ $3.080 \mathrm{GeV}$ [169]. The measured cross-section is maximum right at the $\Lambda \bar{\Lambda}$ threshold and falls off at higher energies contrary to theoretical expectations (see Fig. 6). The cross-section value at threshold is $0.305 \pm 0.058 \mathrm{nb}$, about one half the size of the $0.60 \mathrm{nb}$ jump calculated for a (hypothetical) charged point-like baryon with mass $m_{B}=m_{\Lambda}$. One theoretical analysis concluded that such a threshold jump in the $e^{+} e^{-} \rightarrow \Lambda \bar{\Lambda}$ cross-section is a strong indication of the presence of a very narrow, ${ }^{3} S_{1} \Lambda \bar{\Lambda}$ resonance with a mass close to $2 m_{\Lambda}$ [200].

Measurements of near-threshold $e^{+} e^{-} \rightarrow B \bar{B}$ pair production cross-sections uncovered some intriguing discrepancies with theoretical expectations. To help clarify the underlying physics scenario, BESIII will perform precise cross-section measurements at all of the stable $B \bar{B}$ thresholds that are accessible in the BEPCII CM energy range, including the three $\Sigma \bar{\Sigma}$ and two $\Xi \bar{\Xi}$ thresholds, as well as those for $\Omega^{-} \bar{\Omega}^{+}$and $\Lambda_{c}^{+} \bar{\Lambda}_{c}^{-}$.

\section{OUTLOOK}

Prior to the start of BESIII operation, the BES scientific community, including theorists and experimentalists, prepared an 800-page report that mapped out a diverse ten-year-long physics research program for the project [78]. During its first decade of operation, BESIII has, in accordance with this plan: improved on its measurement of $m_{\tau}$ and $\sigma\left(e^{+} e^{-} \rightarrow\right.$ hadrons); produced world's best measurements of charmed particle decay constants, form-factors and the $\left|V_{c s}\right|$ and $\left|V_{c d}\right|$ CKM matrix elements; reported definitive measurements of the $\eta_{c}$ [201] and $h_{c}$ [202] masses and widths plus many other precision charmonium results; and made a number contributions to the understanding of light hadron physics, such as confirming that the $X(1835)$ structure has a strong coupling to the nucleon-antinucleon final state and identifying strong candidates for the scalar and tensor glueballs.

In addition, during this period, BESIII produced a number of results that were not anticipated in the 2008 plan. These include: discoveries of the $Z_{c}(3900)$ and $Z_{c}(4020)$ charged charmonium-like states; a complex structure of the $Y(4260)$ line shape; $X(3872)$ production via radiative $Y(4260)$ transitions; substantial signals for $a_{0}^{0}(980) \leftrightarrow f_{0}(980)$ mixing; large transverse $\Lambda$ polarization in $J / \psi \rightarrow \Lambda \bar{\Lambda}$ decays and world's best limits on $C P V$ asymmetries in $\Lambda$ hyperon decays; an anomalous threshold behaviour for $\sigma\left(e^{+} e^{-} \rightarrow \Lambda \bar{\Lambda}\right)$ that strongly contradicts theoretical expectations; and measurements of the strong phase in quantum-correlated $D^{0} \rightarrow K^{0} \pi^{+} \pi^{-}$decays.

Each of the items listed in the previous paragraph beg for additional, high-statistics investigations that were not considered in the original BESIII plan. The puzzles associated with the XYZ mesons call for high-statistics data runs that span the 4.0 to $4.7 \mathrm{GeV}$ energy region in small increments to support advanced, $\mathrm{E}_{\mathrm{CM}}$-dependent coupled-channel PWA studies in addition to a unique set of $X(3872)$-related measurements that could be done with a large data set accumulated at the peak energy of the $Y(4260)$ resonance. The $\Lambda$ transverse polarization in $J / \psi \rightarrow \Lambda \bar{\Lambda}$ decays provides singular, and previously unexpected, opportunities to search for new sources of $C P V$ in hyperon decays that could use many times the currently available $J / \psi$ data. Measurements of strong phases in quantum-correlated $D^{0}$ decays with the precision required for the interpretation of future, high profile LHCb and Belle II measurements of the $\gamma C P V$ angle are only possible with BESIII, and will require two or three years of dedicated data-taking at the peak of the $\psi(3770)$. The anomalous $e^{+} e^{-} \rightarrow \Lambda \bar{\Lambda}$ threshold behaviour motivates the accumulation of high-statistics data sets in the vicinities of each of the hyperon-antihyperon thresholds. This menu of measure- 
ments will, at minimum, require operating the program for another decade [203].

In support of this program, the luminosity performance of BEPCII is being upgraded and the maximum CM energy is being increased. In the current mode of operation, the beams are accumulated in the storage rings and then made to collide until the luminosity decreases to about two-thirds of its initial value, at which time collisions are stopped and the beams restored to their maximum currents. A system that is currently being implemented will continuously maintain the beam currents near their peak values, thereby increasing the time-integrated luminosity by about $30 \%$. A two-year-long program that involves upgrading the ring magnet power supplies and replacing some critical magnets will extend the maximum $\mathrm{E}_{\mathrm{CM}}$ from 4.6 to $4.9 \mathrm{GeV}$. This will provide full coverage of the $Y(4660)$, a $1^{--}$charmonium-like resonance that peaks near $\mathrm{E}_{\mathrm{CM}}=4660 \mathrm{MeV}$, improve the production rate of $\Lambda_{c}$ baryons, and provide access to $\Sigma_{c}$ and excited $\Lambda_{c}$ baryon states.

In the longer-term future, two upgraded versions of the BESIII/BEPCII facility, so-called super tau-charm factories, have been proposed: the STCF in China [7] and the SCTF in Russia [8]. Both machines would run at $\mathrm{CM}$ energies that reach $6 \mathrm{GeV}$ or higher, with peak luminosities of $10^{35} \mathrm{~cm}^{-2} \mathrm{~s}^{-1}$, corresponding to a factor of 100 improvement over BEPCII. These improvements would enable systematic studies of charm particle decays, XYZ mesons, searches for new physics sources of $C P V$ in hyperon decays, and the elucidation of many issues in light-hadron physics with unprecedented precision.

\section{Appendix A: The BESIII experiment at BEPCII}

The construction of the Beijing Electron-Positron Collider (BEPC) started in 1984 and the first operation for high-energy physics and synchrotron radiation applications occurred in 1989. In 2008, BEPC was upgraded to BEPCII, a double-ring collider with a peak luminosity of $1.0 \times$ $10^{33} \mathrm{~cm}^{-2} \mathrm{~s}^{-1}$, about two orders of magnitude higher than the maximum achieved with BEPC. For about six months of each year, BEPCII operates at center-of-mass energies that range between 2.0 and $4.6 \mathrm{GeV}$ for particle physics experiments with the Beijing Spectrometer III (BESIII). For three months, it operates in a single beam mode with an energy of $2.5 \mathrm{GeV}$ to provide high fluxes of synchrotron X-rays to 14 separate beam lines for material and biological science applications.

The BESIII experiment [4] recorded its first collisions in July 2008. The detector, shown in Fig. 7, is a general-purpose spectrometer based on a 1 Tesla superconducting (SC) solenoid magnet with a geometrical acceptance that covers $93 \%$ of $4 \pi$ steradians. It consists of a layered arrangement of nested instruments, including: a small cell helium-based multi-layered drift chamber (MDC) that provides momentum measurements of charged particles; a cylinder of plastic scintillators that are used to identify charged particles based on their time-of-flight (ToF); an electromagnetic calorimeter that is a mosaic array of $\mathrm{CsI}(\mathrm{Tl})$ crystals that are used to detect and measure the energies of $\gamma$-rays and provide trigger signals; a surrounding array of Resistive Plate Chambers (RPC) to detect and identify muons. The MDC provides $0.5 \%$ momentum resolution for $1 \mathrm{GeV} / c$ charged particle tracks and energy-loss $(d E / d x)$ measurements with a resolution of $6 \%$. The electromagnetic calorimeter energy resolution for $1 \mathrm{GeV} \gamma$ rays is $2.5 \%$ in the polar angle region $|\cos \theta|<0.83$ (barrel) and $5 \%$ for $0.83 \leq|\cos \theta| \leq 0.93$ (endcaps). The TOF system time resolution is $68 \mathrm{ps}$ in the barrel region and $110 \mathrm{ps}$ in the endcaps. In 2015, the endcap TOF scintillators were replaced with a system of multi-layer RPCs that improved the time resolution to 60 ps [204]. 


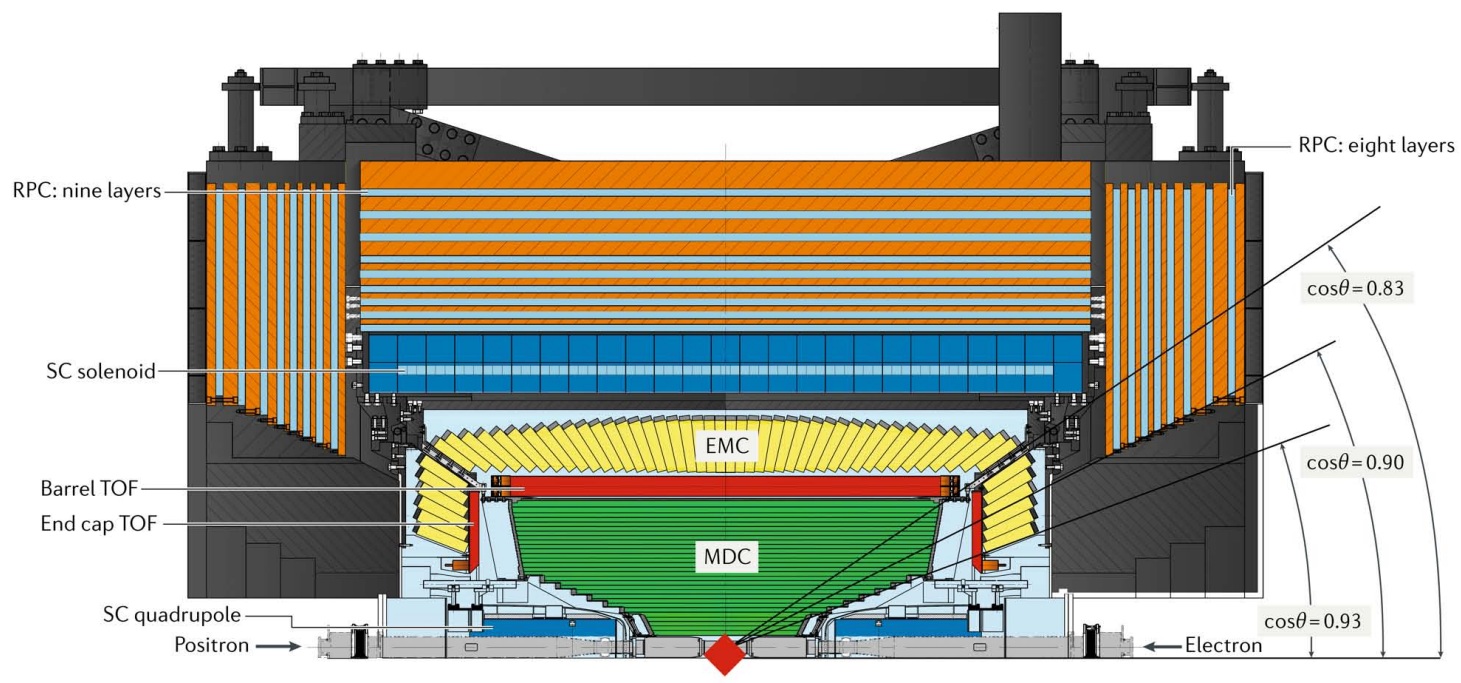

FIG. 7: Cutaway view of the top half of the BESIII detector. (Produced by Dr. Xiao-Yan Ma.)

\section{Acknowledgments}

This work is supported in part by National Natural Science Foundation of China (NSFC) under contract Nos. 11835012, 11521505, and 11475187; the Ministry of Science and Technology of China under Contract No. 2015CB856701; Key Research Program of Frontier Sciences, CAS, Grant No. QYZDJ-SSW-SLH011; the CAS Center for Excellence in Particle Physics (CCEPP); and the CAS President International Fellowship Initiative (PIFI) program.

[1] S. Weinberg, Phys. Rev. Lett. 121, 220001 (2018).

[2] B. Aubert et al. (BaBar), Nucl. Instrum. Meth. A479, 1 (2002), hep-ex/0105044.

[3] A. Abashian et al. (Belle), Nucl. Instrum. Meth. A479, 117 (2002).

[4] M. Ablikim et al. (BESIII), Nucl. Instrum. Meth. A614, 345 (2010), 0911.4960.

[5] A. A. Alves, Jr. et al. (LHCb), JINST 3, S08005 (2008).

[6] W. Altmannshofer et al. (Belle II) (2018), 1808.10567.

[7] Q. Luo and D. Xu, in Proceedings, 9th International Particle Accelerator Conference (IPAC 2018): Vancouver, BC Canada (2018), p. MOPML013.

[8] E. B. Levichev, A. N. Skrinskii, G. M. Tumaikin, and Y. M. Shatunov, Phys. Usp. 61, 405 (2018), URL https://ufn.ru/en/articles/2018/5/b/,

[9] A. J. Bevan et al. (BaBar, Belle), Eur. Phys. J. C74, 3026 (2014), 1406.6311.

[10] M. Kobayashi and T. Maskawa, Prog. Theor. Phys. 49, 652 (1973).

[11] N. Brambilla et al., Eur. Phys. J. C71, 1534 (2011), 1010.5827.

[12] H.-X. Chen, W. Chen, X. Liu, and S.-L. Zhu, Phys. Rept. 639, 1 (2016), 1601.02092.

[13] S. L. Olsen, T. Skwarnicki, and D. Zieminska, Rev. Mod. Phys. 90, 015003 (2018), 1708.04012.

[14] F.-K. Guo, C. Hanhart, U.-G. Meißner, Q. Wang, Q. Zhao, and B.-S. Zou, Rev. Mod. Phys. 90, 015004 (2018), 1705.00141. 
[15] N. Brambilla, S. Eidelman, C. Hanhart, A. Nefediev, C.-P. Shen, C. E. Thomas, A. Vairo, and C.-Z. Yuan (2019), 1907.07583.

[16] M. Gell-Mann, Phys. Lett. 8, 214 (1964).

[17] G. Zweig (1964), An SU(3) model for strong interaction symmetry and its breaking. CERN-TH-401.

[18] J. Z. Bai et al. (BES), Nucl. Instrum. Meth. A344, 319 (1994).

[19] J. Z. Bai et al. (BES), Nucl. Instrum. Meth. A458, 627 (2001).

[20] Q. Qin, L. Ma, J. Wang, and C. Zhang, Conf. Proc. C100523, WEXMH01 (2010).

[21] J. Z. Bai et al. (BES), Phys. Rev. Lett. 69, 3021 (1992).

[22] J. Z. Bai et al. (BES), Phys. Rev. Lett. 74, 4599 (1995).

[23] J. Z. Bai et al. (BES), Phys. Rev. Lett. 88, 101802 (2002), hep-ex/0102003.

[24] M. Ablikim et al. (BES), Phys. Lett. B598, 149 (2004), hep-ex/0406038.

[25] M. Ablikim et al. (BES), Phys. Lett. B633, 681 (2006), hep-ex/0506055.

[26] X.-H. Mo, C.-Z. Yuan, and P. Wang, High Energy Phys. \& Nucl. Phys. 31, 686 (2007), hep$\mathrm{ph} / 0611214$.

[27] R. L. Jaffe, Phys. Rept. 409, 1 (2005), hep-ph/0409065.

[28] W.-J. Deng, H. Liu, L.-C. Gui, and X.-H. Zhong, Phys. Rev. D95, 034026 (2017), 1608.00287.

[29] S. J. Brodsky, D. S. Hwang, and R. F. Lebed, Phys. Rev. Lett. 113, 112001 (2014), 1406.7281.

[30] A. Bondar et al. (Belle), Phys. Rev. Lett. 108, 122001 (2012), 1110.2251.

[31] R. Aaij et al. (LHCb), Phys. Rev. Lett. 115, 072001 (2015), 1507.03414.

[32] M. Ablikim et al. (BESIII), Phys. Rev. D90, 012001 (2014), 1405.1076.

[33] E. V. Abakumova et al., Nucl. Instrum. Meth. A659, 21 (2011), 1109.5771.

[34] J. Y. Zhang (BESIII) (2018), 1812.10056.

[35] G. W. Bennett et al. (Muon g-2), Phys. Rev. D73, 072003 (2006), hep-ex/0602035.

[36] M. Davier, A. Hoecker, B. Malaescu, and Z. Zhang, Eur. Phys. J. C77, 827 (2017), 1706.09436.

[37] A. Keshavarzi, D. Nomura, and T. Teubner, Phys. Rev. D97, 114025 (2018), 1802.02995.

[38] J. Grange et al. (Muon g-2) (2015), 1501.06858.

[39] M. Otani (E34), JPS Conf. Proc. 8, 025008 (2015).

[40] K. Miura, in 36th International Symposium on Lattice Field Theory (Lattice 2018) East Lansing, MI, United States, July 22-28, 2018 (2019), 1901.09052.

[41] C. T. H. Davies et al. (Fermilab Lattice, LATTICE-HPQCD, MILC) (2019), 1902.04223.

[42] M. N. Achasov et al., J. Exp. Theor. Phys. 103, 380 (2006), [Zh. Eksp. Teor. Fiz.130,437(2006)], hep-ex/0605013.

[43] J. P. Lees et al. (BaBar), Phys. Rev. D86, 032013 (2012), 1205.2228.

[44] M. Ablikim et al. (BESIII), Phys. Lett. B753, 629 (2016), 1507.08188.

[45] R. R. Akhmetshin et al. (CMD-2), Phys. Lett. B578, 285 (2004), hep-ex/0308008.

[46] R. R. Akhmetshin et al. (CMD-2), Phys. Lett. B648, 28 (2007), hep-ex/0610021.

[47] A. Anastasi et al. (KLOE-2), JHEP 03, 173 (2018), 1711.03085.

[48] R. R. Akhmetshin et al., Phys. Lett. B768, 345 (2017), 1612.04483.

[49] M. Ablikim et al. (BESIII) (2019), 1912.11208.

[50] M. Ripka (BESIII), Nucl. Part. Phys. Proc. 294-296, 158 (2018).

[51] M. Ablikim et al. (BESIII), Chin. Phys. C41, 063001 (2017), 1702.04977.

[52] A. Bazavov et al., Phys. Rev. D98, 074512 (2018), 1712.09262.

[53] M. Ablikim et al. (BESIII), Phys. Rev. D89, 051104 (2014), 1312.0374.

[54] M. Tanabashi et al. (Particle Data Group), Phys. Rev. D98, 030001 (2018).

[55] M. Ablikim et al. (BESIII), Phys. Rev. Lett. 122, 071802 (2019), 1811.10890.

[56] N. Carrasco et al., Phys. Rev. D91, 054507 (2015), 1411.7908. 
[57] D. Besson et al. (CLEO), Phys. Rev. D80, 032005 (2009), 0906.2983.

[58] M. Ablikim et al. (BESIII), Phys. Rev. D92, 072012 (2015), 1508.07560.

[59] W. Y. Wang, Y. L. Wu, and M. Zhong, Phys. Rev. D67, 014024 (2003), hep-ph/0205157.

[60] M. Ablikim et al. (BESIII), Phys. Rev. Lett. 122, 011804 (2019), 1810.03127.

[61] M. Ablikim et al. (BESIII), Phys. Rev. Lett. 121, 171803 (2018), 1802.05492.

[62] M. Ablikim et al. (BESIII), Phys. Rev. D96, 012002 (2017), 1703.09084.

[63] M. Ablikim et al. (BESIII), Eur. Phys. J. C76, 369 (2016), 1605.00068.

[64] S. Bifani, S. Descotes-Genon, A. Romero Vidal, and M.-H. Schune, J. Phys. G46, 023001 (2019), 1809.06229.

[65] L. Riggio, G. Salerno, and S. Simula, Eur. Phys. J. C78, 501 (2018), 1706.03657.

[66] N. R. Soni and J. N. Pandya, Phys. Rev. D96, 016017 (2017), 1706.01190.

[67] M. Ablikim et al. (BESIII), Phys. Rev. D94, 032001 (2016), 1512.08627.

[68] M. Ablikim et al. (BESIII), Phys. Rev. Lett. 121, 081802 (2018), 1803.02166.

[69] M. Ablikim et al. (BESIII), Phys. Rev. D99, 011103 (2019), 1811.11349.

[70] M. Ablikim et al. (BESIII), Phys. Rev. Lett. 122, 062001 (2019), 1809.06496.

[71] M. Ablikim et al. (BESIII), Phys. Rev. Lett. 122, 061801 (2019), 1811.02911.

[72] M. Ablikim et al. (BESIII), Phys. Rev. D92, 071101 (2015), 1508.00151.

[73] M. Ablikim et al. (BESIII), Phys. Rev. D97, 012006 (2018), 1709.03680.

[74] M. Ablikim et al. (BESIII), Phys. Rev. D92, 112008 (2015), 1510.00308.

[75] M. Ablikim et al. (BESIII), Phys. Rev. D94, 112003 (2016), 1608.06484.

[76] L.-L. Chau and W.-Y. Keung, Phys. Rev. Lett. 53, 1802 (1984).

[77] Y. Amhis et al. (HFLAV) (2018), 1812.07461.

[78] D. M. Asner et al., Int. J. Mod. Phys. A24, S1 (2009), 0809.1869.

[79] J. Libby et al. (CLEO), Phys. Rev. D82, 112006 (2010), 1010.2817.

[80] R. Aaij et al. (LHCb), JHEP 08, 176 (2018), [JHEP18,176(2020)], 1806.01202.

[81] S. S. Malde, Tech. Rep. LHCb-PUB-2016-025. CERN-LHCb-PUB-2016-025, CERN, Geneva (2016), URL http://cds.cern.ch/record/2223391.

[82] G. S. Abrams et al., Phys. Rev. Lett. 44, 10 (1980).

[83] A. Zupanc et al. (Belle), Phys. Rev. Lett. 113, 042002 (2014), 1312.7826.

[84] M. Ablikim et al. (BESIII), Phys. Rev. Lett. 115, 221805 (2015), 1510.02610.

[85] M. Ablikim et al. (BESIII), Phys. Lett. B767, 42 (2017), 1611.04382.

[86] M. Ablikim et al. (BESIII), Phys. Rev. Lett. 116, 052001 (2016), 1511.08380.

[87] M. Ablikim et al. (BESIII), Phys. Rev. Lett. 120, 132001 (2018), 1710.00150.

[88] G. Pakhlova et al. (Belle), Phys. Rev. Lett. 101, 172001 (2008), 0807.4458.

[89] X. L. Wang et al. (Belle), Phys. Rev. Lett. 99, 142002 (2007), 0707.3699.

[90] M. Ablikim et al. (BESIII), Phys. Rev. Lett. 110, 252001 (2013), 1303.5949.

[91] Z. Q. Liu et al. (Belle), Phys. Rev. Lett. 110, 252002 (2013), 1304.0121.

[92] T. Xiao, S. Dobbs, A. Tomaradze, and K. K. Seth, Phys. Lett. B727, 366 (2013), 1304.3036.

[93] M. Ablikim et al. (BESIII), Phys. Rev. Lett. 111, 242001 (2013), 1309.1896.

[94] M. Ablikim et al. (BESIII), Phys. Rev. Lett. 118, 092001 (2017), 1611.01317.

[95] M. Ablikim et al. (BESIII), Phys. Rev. Lett. 112, 092001 (2014), 1310.4101.

[96] M. Ablikim et al. (BESIII), Phys. Rev. Lett. 119, 072001 (2017), 1706.04100.

[97] M. Ablikim et al. (BESIII), Phys. Rev. Lett. 112, 022001 (2014), 1310.1163.

[98] M. Ablikim et al. (BESIII), Phys. Rev. D92, 092006 (2015), 1509.01398.

[99] C.-Z. Yuan, Int. J. Mod. Phys. A33, 1830018 (2018), 1808.01570.

[100] M. Ablikim et al. (BESIII), Phys. Rev. D100, 111102 (2019), 1906.00831. 
[101] M. Ablikim et al. (BESIII), Phys. Rev. D96, 032004 (2017), 1703.08787.

[102] S. K. Choi et al. (Belle), Phys. Rev. Lett. 91, 262001 (2003), hep-ex/0309032.

[103] N. A. Tornqvist (2003), hep-ph/0308277.

[104] Q. Wang, C. Hanhart, and Q. Zhao, Phys. Rev. Lett. 111, 132003 (2013), 1303.6355.

[105] L. Maiani, V. Riquer, R. Faccini, F. Piccinini, A. Pilloni, and A. D. Polosa, Phys. Rev. D87, 111102 (2013), 1303.6857.

[106] R. F. Lebed and A. D. Polosa, Phys. Rev. D93, 094024 (2016), 1602.08421.

[107] M. Ablikim et al. (BESIII), Phys. Rev. Lett. 112, 132001 (2014), 1308.2760.

[108] D. V. Bugg, EPL 96, 11002 (2011), 1105.5492.

[109] D.-Y. Chen, X. Liu, and T. Matsuki, Phys. Rev. D88, 036008 (2013), 1304.5845.

[110] E. S. Swanson, Int. J. Mod. Phys. E25, 1642010 (2016), 1504.07952.

[111] F.-K. Guo, C. Hanhart, Q. Wang, and Q. Zhao, Phys. Rev. D91, 051504 (2015), 1411.5584.

[112] A. P. Szczepaniak, Phys. Lett. B757, 61 (2016), 1510.01789.

[113] M. Ablikim et al. (BESIII), Phys. Rev. Lett. 115, 112003 (2015), 1506.06018.

[114] M. Ablikim et al. (BESIII), Phys. Rev. Lett. 115, 222002 (2015), 1509.05620.

[115] M. Ablikim et al. (BESIII), Phys. Rev. Lett. 113, 212002 (2014), 1409.6577.

[116] M. Ablikim et al. (BESIII), Phys. Rev. Lett. 115, 182002 (2015), 1507.02404.

[117] B. Aubert et al. (BaBar), Phys. Rev. Lett. 95, 142001 (2005), hep-ex/0506081.

[118] B. Aubert et al. (BaBar), Phys. Rev. Lett. 98, 212001 (2007), hep-ex/0610057.

[119] C.-F. Qiao, Phys. Lett. B639, 263 (2006), hep-ph/0510228.

[120] G.-J. Ding, Phys. Rev. D79, 014001 (2009), 0809.4818.

[121] L. Y. Dai, M. Shi, G.-Y. Tang, and H. Q. Zheng, Phys. Rev. D92, 014020 (2015), 1206.6911.

[122] L. Maiani, V. Riquer, F. Piccinini, and A. D. Polosa, Phys. Rev. D72, 031502 (2005), hep$\mathrm{ph} / 0507062$.

[123] A. Ali, L. Maiani, A. V. Borisov, I. Ahmed, M. Jamil Aslam, A. Ya. Parkhomenko, A. D. Polosa, and A. Rehman, Eur. Phys. J. C78, 29 (2018), 1708.04650.

[124] S.-L. Zhu, Phys. Lett. B625, 212 (2005), hep-ph/0507025.

[125] E. Kou and O. Pene, Phys. Lett. B631, 164 (2005), hep-ph/0507119.

[126] S. Dubynskiy and M. B. Voloshin, Phys. Lett. B666, 344 (2008), 0803.2224.

[127] C. Z. Yuan et al. (Belle), Phys. Rev. Lett. 99, 182004 (2007), 0707.2541.

[128] C. Patrignani et al. (Particle Data Group), Chin. Phys. C40, 100001 (2016).

[129] M. Ablikim et al. (BESIII), Phys. Rev. Lett. 118, 092002 (2017), 1610.07044.

[130] M. Ablikim et al. (BESIII), Phys. Rev. Lett. 114, 092003 (2015), 1410.6538.

[131] M. Ablikim et al. (BESIII), Phys. Rev. Lett. 122, 102002 (2019), 1808.02847.

[132] M. Ablikim et al. (BESIII) (2019), 1901.03992.

[133] S. Dubynskiy and M. B. Voloshin, Phys. Rev. D77, 014013 (2008), 0709.4474.

[134] M. Ablikim et al. (BESIII) (2019), 1903.04695.

[135] R. L. Jaffe and K. Johnson, Phys. Lett. 60B, 201 (1976).

[136] W. Toki, in The Strong interaction, from hadrons to partons: Proceedings, 24th SLAC Summer Institute on Particle Physics (SSI 96), Stanford, Calif., 19-30 Aug 1996 (1996), pp. 141-169, URL http://www.slac.stanford.edu/pubs/confproc/ssi96/ssi96-006.html.

[137] Y. Chen et al., Phys. Rev. D73, 014516 (2006), hep-lat/0510074.

[138] M. Ablikim et al. (BESIII), Phys. Rev. D92, 052003 (2015), [Erratum: Phys. Rev. D93, no.3, 039906 (2016)], 1506.00546.

[139] M. Ablikim et al. (BESIII), Phys. Rev. D98, 072003 (2018), 1808.06946.

[140] M. Ablikim et al. (BESIII), Phys. Rev. D87, 092009 (2013), [Erratum: Phys. Rev. D87, no.11, 
119901 (2013)], 1301.0053.

[141] L.-C. Gui, Y. Chen, G. Li, C. Liu, Y.-B. Liu, J.-P. Ma, Y.-B. Yang, and J.-B. Zhang (CLQCD), Phys. Rev. Lett. 110, 021601 (2013), 1206.0125.

[142] M. Ablikim et al. (BESIII), Phys. Rev. D93, 112011 (2016), 1602.01523.

[143] Y.-B. Yang, L.-C. Gui, Y. Chen, C. Liu, Y.-B. Liu, J.-P. Ma, and J.-B. Zhang (CLQCD), Phys. Rev. Lett. 111, 091601 (2013), 1304.3807.

[144] J. Z. Bai et al. (BES), Phys. Rev. Lett. 91, 022001 (2003), hep-ex/0303006.

[145] M. Ablikim et al. (BESIII), Phys. Rev. Lett. 108, 112003 (2012), 1112.0942.

[146] M. Ablikim et al. (BES), Phys. Rev. Lett. 95, 262001 (2005), hep-ex/0508025.

[147] G.-J. Ding and M.-L. Yan, Phys. Rev. C72, 015208 (2005), hep-ph/0502127.

[148] M. Ablikim et al. (BESIII), Phys. Rev. Lett. 117, 042002 (2016), 1603.09653.

[149] M. Ablikim et al. (BESIII), Phys. Rev. Lett. 107, 182001 (2011), 1107.1806.

[150] M. Ablikim et al. (BESIII), Phys. Rev. D88, 091502 (2013), 1305.5333.

[151] M. Ablikim et al. (BESIII), Phys. Rev. D87, 112004 (2013), 1303.3108.

[152] M. Ablikim et al. (BESIII), Phys. Rev. D93, 052010 (2016), 1512.08197.

[153] M. Battaglieri et al., Acta Phys. Polon. B46, 257 (2015), 1412.6393.

[154] R. Armenteros, D. N. Edwards, and T. Jacobsen, Phys. Lett. 17, 344 (1965).

[155] S. D. Protopopescu, M. Alston-Garnjost, A. Barbaro-Galtieri, S. M. Flatte, J. H. Friedman, T. A. Lasinski, G. R. Lynch, M. S. Rabin, and F. T. Solmitz, Phys. Rev. D7, 1279 (1973).

[156] N. N. Achasov, Phys. Part. Nucl. 48, 681 (2017).

[157] J. D. Weinstein and N. Isgur, Phys. Rev. D41, 2236 (1990).

[158] R. L. Jaffe, Phys. Rev. D15, 267 (1977).

[159] D. Black, A. H. Fariborz, F. Sannino, and J. Schechter, Phys. Rev. D59, 074026 (1999), hep$\mathrm{ph} / 9808415$.

[160] J. A. Oller and E. Oset, Phys. Rev. D60, 074023 (1999), hep-ph/9809337.

[161] L. Maiani, F. Piccinini, A. D. Polosa, and V. Riquer, Phys. Rev. Lett. 93, 212002 (2004), hep$\mathrm{ph} / 0407017$.

[162] G. 't Hooft, G. Isidori, L. Maiani, A. D. Polosa, and V. Riquer, Phys. Lett. B662, 424 (2008), 0801.2288.

[163] S. Ishida, M. Y. Ishida, H. Sawazaki, K. Yamada, T. Ishida, T. Kinashi, K. Takamatsu, and T. Tsuru, in Meson nucleon physics and the structure of the nucleon. Proceedings, 6th International Symposium, Blaubeuren, Germany, July 10-14, 1995. Vols. 1, 2 (1995), pp. 454-456.

[164] N. N. Achasov, S. A. Devyanin, and G. N. Shestakov, Phys. Lett. 88B, 367 (1979).

[165] M. Ablikim et al. (BESIII), Phys. Rev. Lett. 121, 022001 (2018), 1802.00583.

[166] A. Z. Dubnickova, S. Dubnicka, and M. P. Rekalo, Nuovo Cim. A109, 241 (1996).

[167] G. Fäldt and A. Kupsc, Phys. Lett. B772, 16 (2017), 1702.07288.

[168] M. Ablikim et al. (BESIII) (2018), 1808.08917.

[169] M. Ablikim et al. (BESIII), Phys. Rev. D97, 032013 (2018), 1709.10236.

[170] S. Pacetti, R. Baldini Ferroli, and E. Tomasi-Gustafsson, Phys. Rept. 550-551, 1 (2015).

[171] J. W. Cronin and O. E. Overseth, Phys. Rev. 129, 1795 (1963).

[172] O. E. Overseth and R. F. Roth, Phys. Rev. Lett. 19, 391 (1967).

[173] P. M. Dauber, J. P. Berge, J. R. Hubbard, D. W. Merrill, and R. A. Muller, Phys. Rev. 179, 1262 (1969).

[174] W. E. Cleland, G. Conforto, G. H. Eaton, H. J. Gerber, M. Reinharz, A. Gautschi, E. Heer, C. Revillard, and G. Von Dardel, Nucl. Phys. B40, 221 (1972).

[175] P. Astbury et al., Nucl. Phys. B99, 30 (1975). 
[176] P. D. Barnes et al., Phys. Rev. C54, 1877 (1996).

[177] J. F. Donoghue, X.-G. He, and S. Pakvasa, Phys. Rev. D34, 833 (1986).

[178] S. Olsen, L. Pondrom, R. Handler, P. Limon, J. A. Smith, and O. E. Overseth, Phys. Rev. Lett. 24, 843 (1970).

[179] E. Gonzalez and J. I. Illana, in Tau charm factory. Proceedings, 3rd Workshop, Marbella, Spain, June 1-6, 1993 (1994), pp. 525-538.

[180] D. E. Morrissey and M. J. Ramsey-Musolf, New J. Phys. 14, 125003 (2012), 1206.2942.

[181] T. Aushev et al. (2010), 1002.5012.

[182] I. Bediaga et al. (LHCb) (2012).

[183] R. Aaij et al. (LHCb) (2018), 1808.08865.

[184] R. Acciarri et al. (DUNE) (2016), 1601.05471.

[185] K. Abe et al. (Hyper-Kamiokande) (2018), 1805.04163.

[186] R. Aaij et al. (LHCb) (2019), 1903.08726.

[187] A. Khodjamirian and A. A. Petrov, Phys. Lett. B774, 235 (2017), 1706.07780.

[188] M. Golden and B. Grinstein, Phys. Lett. B222, 501 (1989).

[189] F. Buccella, M. Lusignoli, G. Miele, A. Pugliese, and P. Santorelli, Phys. Rev. D51, 3478 (1995), hep-ph/9411286.

[190] S. Bianco, F. L. Fabbri, D. Benson, and I. Bigi, Riv. Nuovo Cim. 26N7, 1 (2003), hep-ex/0309021.

[191] Y. Grossman, A. L. Kagan, and Y. Nir, Phys. Rev. D75, 036008 (2007), hep-ph/0609178.

[192] A. D. Sakharov, Zh. Eksp. Teor. Fiz. 18, 631 (1948), [Usp. Fiz. Nauk161,no.5,29(1991)].

[193] A. B. Arbuzov and T. V. Kopylova, JHEP 04, 009 (2012), 1111.4308.

[194] J. P. Lees et al. (BaBar), Phys. Rev. D87, 092005 (2013), 1302.0055.

[195] R. R. Akhmetshin et al. (CMD-3) (2018), 1808.00145.

[196] A. Antonelli et al. (FENICE), Nucl. Phys. B517, 3 (1998).

[197] M. N. Achasov et al. (SND), Phys. Rev. D90, 112007 (2014), 1410.3188.

[198] D. Bisello et al. (DM2), Z. Phys. C48, 23 (1990).

[199] B. Aubert et al. (BaBar), Phys. Rev. D76, 092006 (2007), 0709.1988.

[200] J. Haidenbauer and U. G. Meißner, Phys. Lett. B761, 456 (2016), 1608.02766.

[201] M. Ablikim et al. (BESIII), Phys. Rev. Lett. 108, 222002 (2012), 1111.0398.

[202] M. Ablikim et al. (BESIII), Phys. Rev. D86, 092009 (2012), 1209.4963.

[203] M. Ablikim et al. (BESIII) (2019), 1912.05983.

[204] X. Wang et al., JINST 11, C08009 (2016), 1604.02701. 